\title{
The Incremental Cooperative Design of Preventive Healthcare Networks
}

\author{
Soheil Davari* \\ University of Hertfordshire, Hertfordshire Business School \\ United Kingdom
}

\begin{abstract}
In the Preventive Healthcare Network Design Problem (PHNDP), one seeks to locate facilities in a way that the uptake of services is maximised given certain constraints such as congestion considerations. We introduce the incremental and cooperative version of the problem, IC-PHNDP for short, in which facilities are added incrementally to the network (one at a time), contributing to the service levels. We first develop a general non-linear model of this problem and then present a method to make it linear. As the problem is of a combinatorial nature, an efficient Variable Neighbourhood Search (VNS) algorithm is proposed to solve it. In order to gain insight into the problem, the computational studies were performed with randomly generated instances of different settings. Results clearly show that VNS performs well in solving IC-PHNDP with errors not more than $1.54 \%$.
\end{abstract}

Keywords. Preventive healthcare, Facility location, Cooperative covering, Variable neighbourhood search, Network design.

\section{Introduction}

Limited resources and ageing population are two of the major challenges of the health industry in the 21st century. The problem of managing healthcare supply chains becomes more complicated by increased customer expectations, shortage of healthcare workers, and the need to invest on new technologies. This has brought about increased healthcare expenditure in many countries. For instance, according to the Office of National Statistics [1], the total healthcare expenditure in the UK as a percentage of gross domestic product had increased from $6.2 \%$ in 1997 to $8.8 \%$ in 2013 .

Preventive healthcare aims at saving lives and improving health through early detection of diseases. It is comprised of programmes such as cholesterol screening, HIV screenings, immunisation vaccination, and diet counseling services. These programmes can prevent a wide range of chronic diseases such as heart disease, cancer, and diabetes which are responsible for seven out of ten deaths and account for $75 \%$ of nation's health spending among Americans [2]. Although most of these services are offered for no cost in most countries, the participation rates are low and improving the uptake rates of these services is a concern to governments all over the world. The uptake rate can be different among various groups of education and occupation (Damiani et al. [3]), income (Fox and Shaw [4]), and gender (Meissner et al. [5]) and a variety of qualitative and quantitative factors can influence it. For instance, the proximity of the service centres, congestion in facilities, and even closeness of these centres to other facilities such as shopping malls can all influence the uptake rates (Refer to Santos et al. [6] and references therein for further information). Among these, proximity to the facilities has been known to be the most significant factor (refer to Muller et al. [7], Varkevisser et al. [8], and Haynes et al. $[9])$.

The current paper deals with a discrete, incremental, and cooperative version of Preventive Healthcare

*Corresponding author Email: s.davari@herts.ac.uk 
Network Design problem (IC-PHNDP) which concerns with finding the optimal number and location of facilities among a set of potential nodes in order to maximise the uptake of services. We assume that the network is built gradually and over the periods (incrementally) and once a facility is opened, it should remain operational until the last period. Similar to any other real-world optimisation problem, it has a set of constraints such as the congestion constraint. There is an analogy between our problem and the competitive facility location problem in the sense that both make use of the idea of gravity-based attraction (Hotelling [10]) to model the attraction of clients to facilities. In this model, the probability of a customer patronizing a facility is proportional to the attractiveness of the facility and inversely proportional with the distance. This idea was later extended by many scientists such as Nakanishi and Cooper [11]. A review of these contributions can be found in Bell et al. [12].

We assume that facilities cooperate in providing services to the clients (in line with the seminal paper of Berman et al. [13]). In such a setting, the coverage of a demand node is not determined by only the closest facility, but all the facilities in its vicinity. In other words, each facility $j$ emits signals decaying over distance based on a known non-negative and non-increasing function of distance $\phi(d(i, j))\left(\right.$ e.g. $\phi(d(i, j))=\frac{1}{d_{i j}^{2}}$ or $\left.\phi(d(i, j))=\exp \left(-d_{i j}\right)\right)$ and each demand point $i$ is affected by an aggregation of all the signals received. This aggregation operator can take different forms such as summation, maximum, and truncated sum (Figure (1) ). Regardless of the coverage type used, a demand point is covered if the aggregated signal exceeds a certain threshold $\Theta$. For instance, in the case of a summation operator and assuming $p$ established facilities, demand point $i$ is covered if and only if:

$$
\sum_{j=1}^{p} \phi(d(i, j)) \geq \Theta
$$
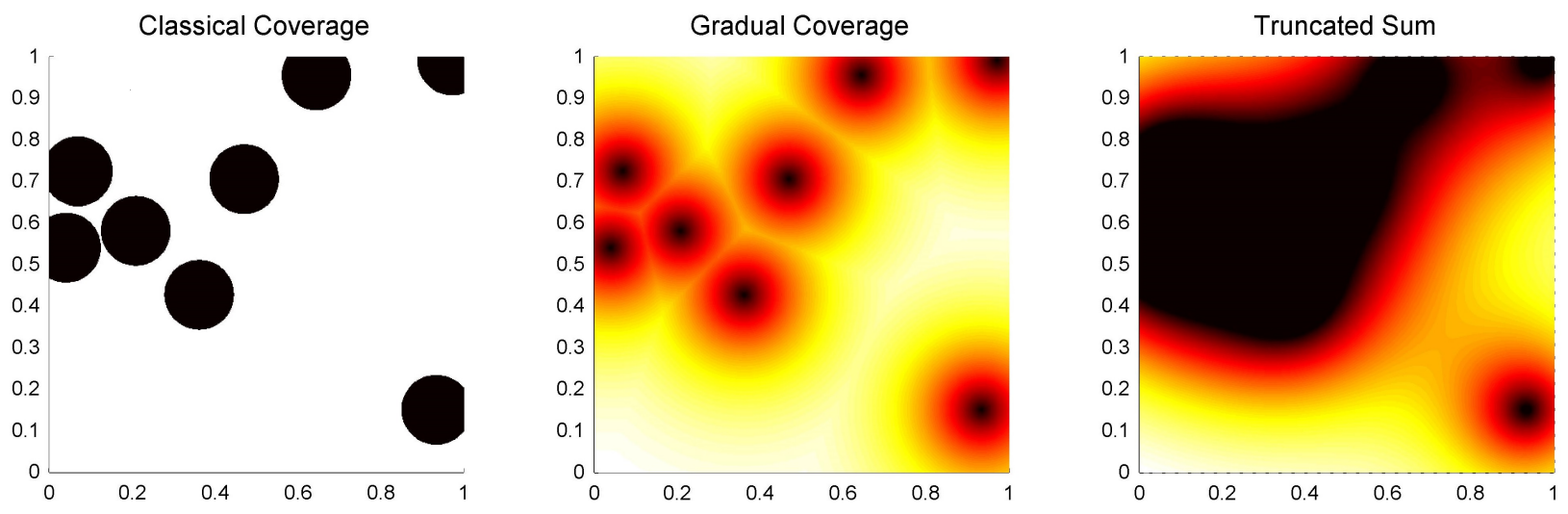

Figure 1: Heat map of different aggregation operators

In this paper, we model IC-PHNDP as a mixed-integer programming model and provide a method to linearize it. Then, we propose an efficient Variable Neighbourhood Search heuristic to solve it with optimality gaps of not more than $1.54 \%$ while the average optimality gap is $0.71 \%$. The contributions of this paper are as follows:

- To the best of our knowledge, there is no publication in the literature for modelling the incremental or cooperative version of a preventive healthcare network design. This paper contributes to the literature by modelling this problem.

- We propose an efficient heuristic procedure to solve the problem and analyse its performance with a set of hypothetically generated test problems.

The outline of this paper is as follows: It proceeds with a literature review of relevant publications in section (2). The mathematical model of the paper is presented in section (3). In section (4), our proposed solution 
procedure is elaborated. Numerical experiments and some analysis appear in section (5), and finally, conclusions and some future research avenues are provided in section (6).

\section{Background and Literature Review}

To the best of our knowledge, Hakimi [14] was the first scholar addressing the problem of healthcare network design in the literature. Later, a lot of research has been carried out on different problems in the area of healthcare network design such as public healthcare facility location (Kim and Kim [15]), health care facility location-allocation (Syam and Cote [16]), and healthcare facility location/vehicle routing (Veenstra et al. [17]). Interested readers can refer to a recent survey by Ahmadi et al. [18] for further information about the healthcare facility location literature.

One of the relatively less studied variants of the healthcare network design is the problem of designing preventive healthcare networks. As far as we know, Verter and LaPierre [19] was the first publication in the literature addressing this problem where case studies in Georgia, USA and Montreal, Canada were given. Following on from that, Zhang et al. [20] presented the problem of preventive healthcare network design on a graph with optimal choice allocation aiming at maximising service uptake and compared the performance of four heuristics in terms of their accuracy and computational requirements. Zhang et al. [21] studied a different version of the problem where a bi-level non-linear optimisation model was developed with equilibrium constraints and a tabu search heuristic was proposed to solve it. Another study in the area of PHNDP is Gu et al. [22] in which the impact of client choice behaviour on the network was studied as a bi-objective model which was solved using an interchange algorithm. Zhang et al. [23] was another study of the client choice behaviour on the network presenting both an optimal choice model and a probabilistic choice model. The problem was formulated as a mixed integer programming problem and a genetic algorithm was presented to solve it. The bi-objective fuzzy variant of the problem was studied in Davari et al. [24] where a fuzzy goal programming and a chance constrained solution procedure were proposed to solve the problem. Haas and Muller [25] employed a multinomial logit model to model the client choice behaviour and solved the problem with instances up to 20 potential nodes and 400 demand zones. They presented a procedure for finding lower bounds for larger sizes and a definition of clients' utility. Davari et al. [26] considered PHNDP with impatient clients and budget constraints and proposed an efficient VNS heuristic to solve it.

The problem of multi-period facility location is not new to the literature and there has been numerous publications dealing with this problem. Table (1) gives an overview of the recent research on multi-period models. Although this table does not cover an exhaustive list of features of each paper, it mainly aims at linking our study to the literature and providing an overview of the solution procedures used in the literature. 
Table 1: Literature review of some recent multi-period facility location models

\begin{tabular}{|c|c|c|c|}
\hline Paper & Year & Problem & Solution approach \\
\hline Gen and Syarif [27] & 2005 & Production/distribution planning & Genetic algorithm \\
\hline McKendall and Shang [28] & 2006 & Dynamic facility layout & Simulated annealing \\
\hline Ko and Evans [29] & 2007 & Integrated forward/reverse logistics network for 3PLs & Genetic algorithm \\
\hline Yi and Ozdamar [30] & 2007 & Evacuation and support in disaster response & Exact method \\
\hline Ndiaye and Alfares [31] & 2008 & Health services for moving population groups & Exact method \\
\hline Wang et al. [32] & 2008 & Two-echelon integrated competitive/Uncompetitive facility location problem & Genetic algorithm \\
\hline Rajagopalan et al. [33] & 2008 & Dynamic redeployment of ambulances & Tabu search \\
\hline Manzini et al. [34] & 2008 & Multi-stage, multi-commodity location allocation & Exact method \\
\hline Gourdin and Klopfenstein [35] & 2008 & Capacitated location with modular equipments & Polyhedral properties \\
\hline Hinojosa et al. $[36]$ & 2008 & Dynamic supply chain design with inventory & Lagrangian relaxation \\
\hline Albareda-Sambola et al. [37] & 2009 & Multi-period incremental service facility location problem & Lagrangian relaxation \\
\hline Lee and Dong $[38]$ & 2009 & Dynamic location and allocation models & Heuristic method \\
\hline Mahar et al. [39] & 2009 & On-line fulfilment assignment problem & Branch \& bound, Dynamic programming \\
\hline Schmid and Doerner [40] & 2010 & Ambulance location-relocation problems with time-dependent travel times & Variable neighbourhood search \\
\hline Basar et al. [41] & 2011 & Emergency medical stations & Tabu search \\
\hline Fazel Zarandi et al. [42] & 2011 & Large-scale dynamic maximal covering location problem & Simulated annealing \\
\hline Torres and Uster [43] & 2011 & Capacitated facility location with relocations and changing demand & Lagrangian relaxation \\
\hline Beneyyan et al. [44] & 2012 & Single and multi-period location-allocation models in the health sector & Exact method \\
\hline Sha and Huang [45] & 2012 & Emergency blood supply scheduling model & Heuristic based on Lagrangian relaxation \\
\hline Rottkemper et al. [46] & 2012 & Inventory relocation and distribution in humanitarian logistics & Rolling horizon solution method \\
\hline Schmid $[47]$ & 2012 & Dynamic ambulance relocation and dispatching problem & Approximate dynamic programming \\
\hline Albareda-Sambola et al. [48] & 2012 & Multi-period Location-Routing with Decoupled Time Scales & Heuristic \\
\hline Albareda-Sambola et al. [49] & 2013 & Multi-period location-allocation problem under uncertainty & Fix-and-Relax-Coordination \\
\hline Ghaderi and Jabalameli [50] & 2013 & Budget-constrained dynamic uncapacitated facility location network design & Exact method and simulated annealing \\
\hline Correia et al. [51] & 2013 & Two-echelon supply chain network design problem with sizing decisions & Valid inequalities \\
\hline Zhen et al. [52] & 2014 & Emergency medical stations & Genetic algorithm \\
\hline Gelareh et al. [53] & 2015 & Multi-period hub location problem & Benders decomposition \\
\hline Chung and Kwon [54] & 2015 & Location of electric car charging station & Heuristic (myopic methods) \\
\hline Elbek and Wohlk [55] & 2016 & Scheduling of recyclable materials collection & Constructive variable neighbourhood search \\
\hline Duhamel et al. [56] & 2016 & Location-allocation problem for post-disaster operations & Decomposition approach \\
\hline Correia and Melo [57] & 2016 & Facility location under delayed demand satisfaction & Valid inequalities \\
\hline Markovic et al. [58] & 2016 & Stochastic facility location problem with independent demand & Lagrangian relaxation \\
\hline Vatsa and Jayaswal [59] & 2016 & Multi-period maximal covering facility location problem with server uncertainty & Benders decomposition \\
\hline
\end{tabular}


From the above and to the best of our knowledge, there is not any attempt in the literature towards modelling the multi-period design of a preventive healthcare network. Another gap in the literature of PHNDP is the realistic assumption of cooperativeness in covering nodes in a preventive helathcare setting. In line with some of the existing literature, our paper aims at filling these two gaps by proposing an efficient integer programming model and presenting an efficient variable neighbourhood search procedure which is capable of solving large instances with errors not worse than $1.54 \%$ to the best known solutions.

\section{Mathematical model}

Consider a region (say a city) with a set of nodes representing demands in different sub-regions. The decision maker is interested in minimising the costs while ensuring that a minimum level of coverage is guaranteed for all the nodes. There are candidate locations to establish facilities and the proximity of clients to facilities is the key factor in making facilities more attractive. Besides, there are congestion considerations in the problem. We assume that there is no existing facility in the network; however, the model is easily generalizable for the case where facilities exist. Moreover, facilities cooperate in providing service to the population centres and the network is established incrementally.

The model is studied in a discrete space with $N$ as the demand nodes $(|N|=m)$ and a set of $V \subset N$ to be the set of potential nodes to establish a facility. The demand associated with node $i \in N$ at time $t \in T$ is denoted as $p_{i t}$. The shortest path between each demand zone $i \in N$ and a potential facility at node $j \in V$ is represented as $d_{i j}$. Moreover, we follow a similar approach to Pastor [60] in defining the attractiveness. Like that, the attractiveness of each facility $j \in V$ to clients living at node $i \in N$ is shown as $\phi_{i j}$ and found using a negative exponential function as $\phi_{i j}=e^{-\eta d_{i j}}$ where $\eta$ is an empirically defined value (different decay functions can be used such as the power function $\phi_{i j}=d^{-\eta}$. However, based on the empirical study of Drezner [61], we use the exponential function in this study). It should be noted that this attractiveness measure can be modified in order to address other attractiveness parameters such as the appearance of a facility, its size, and other factors (see Drezner [62] and references therein). Moreover, in each period $t \in T$, a minimum of $\pi_{t}$ people should be covered. The $\pi$ function is defined as a non-decreasing function of $t$ (linear, piecewise, etc.) to gradually increase the service level in the network.

We assume that the demand of node $j \in V$ is partially met by each opened facility and inversely proportional to the distance between the demand node and the facility (based on the basic concept of gravity rule by Reily [63]). Last but not least, the cooperative aggregation operator is shown as $\Phi_{i t}$ which can take different forms. In this paper, we assume that the aggregate coverage of a node $i$ at time $t\left(\Phi_{i t}\right)$ is found as:

$$
\Phi_{i t}=\min \left\{1, \sum_{j \in V} \phi_{i j} x_{j t}\right\}
$$

where $x_{j t}$ is defined as follows:

$$
x_{j t}= \begin{cases}1 & \text { If there is a facility at node } j \in V \text { at time } t \in T \\ 0 & \text { Otherwise }\end{cases}
$$

The other parameters of the problem are as follows:

$\underline{\text { Parameters }}$ 
$a_{i j} \quad$ The attractiveness of facility at node $j \in V$ to the demand node at node $i \in N$

$d_{i j} \quad$ The distance between nodes $i \in N$ and $j \in V$

$p_{i t} \quad$ The population of node $i \in N$ at time $t \in T$

$f_{j t} \quad$ Cost of establishing a facility at node $j \in V$ at time $t \in T$

$o_{j t} \quad$ Operation cost of node $j \in V$ at time $t \in T$

$\pi_{t} \quad$ The total population to be covered at time $t \in T$

$\bar{\lambda} \quad$ The maximum number of clients each server can serve

$T_{\max } \quad$ Number of periods of the study

Let $\zeta_{i j t}$ to be the share of the facility at node $j \in V$ from the demand at node $i \in N$ at time $t \in T$. Spatial interaction models assume that this ratio equals the relative utility of facility at node $j \in V$ compared to other facilities available on the network which can be represented as follows.

$$
\zeta_{i j t}=\frac{\phi_{i j} x_{j t}}{\sum_{l \in V} \phi_{i l} x_{l t}+\epsilon}
$$

where a sufficiently small $\epsilon$ is added to the denominator to avoid undefined values for $\zeta$. Now, the problem can be formulated as follows.

$$
\begin{array}{lr}
\min _{j \in V} \sum_{t \in T} f_{j t}\left(x_{j t}-x_{j(t-1)}\right)+\sum_{j \in V} \sum_{t \in T} o_{j t} x_{j t} & \\
\Phi_{i t} \leq 1 & \forall i \in N, \forall t \in T \\
\Phi_{i t} \leq \sum_{j \in V} \phi_{i j} x_{j t} & \forall i \in N, \forall t \in T \\
x_{j t} \leq x_{j(t+1)} & \forall j \in V, t \in T \backslash\left\{T_{\text {max }}\right\} \\
\sum_{i \in N} \frac{\phi_{i j} x_{j t}}{\sum_{l \in V} \phi_{i l} x_{l t}+\epsilon} p_{i t} \leq \bar{\lambda} x_{j t} & \forall j \in V, \forall t \in T \\
\sum_{i \in N} \Phi_{i t} p_{i t} \geq \pi_{t} & \\
x_{j t} \in\{0,1\} & \forall j \in V, \forall t \in T
\end{array}
$$

The objective function (4) minimises the total cost of the network which is a function of fixed establishment costs and the variable server costs. Constraints (5) and (6) linearize the equation (2). Constraint set (7) ensures that while a facility is opened, it should operate for the remaining periods. In other words, the problem is modelled as an uninterrupted facility location problem which is a more realistic one in practice. The congestion constraint is enforced to the model through Constraint (8). Constraint (9) states that in each period $t \in T$, a minimum population of $\pi_{t}$ should be covered. As stated earlier, the $\pi$ function can be defined as a non-decreasing function of $t \in T$ to gradually improve the service level in the network. Finally, constraint (10) is the integrality constraint for the variable $x_{j t}$. The model is a non-linear one owing to constraint (8) which can be linearized using Proposition (1).

Proposition 1. Constraint (8) can be rewritten as:

$$
\sum_{i \in N} p_{i t} \phi_{i j} z_{i j t} \leq \bar{\lambda} x_{j t} \quad \forall j \in V, \forall t \in T
$$

Proof. Define the auxiliary variable $w_{i t}$ to represent the following component of constraint (8):

$$
w_{i t}=\frac{1}{\sum_{l \in V} \phi_{i l} x_{l t}+\epsilon}
$$


and the variable $z_{i j t}$ to represent the following component of constraint (8).

$$
z_{i j t}=w_{i t} x_{j t}
$$

Note that there will be a need to add the following constraints to the model.

$$
\begin{array}{lr}
z_{i j t} \leq M x_{j t} & \forall i \in N, \forall j \in V, \forall t \in T \\
z_{i j t} \leq w_{i t} & \forall i \in N, \forall j \in V, \forall t \in T \\
w_{i t}-z_{i j t} \leq M\left(1-x_{j t}\right) & \forall i \in N, \forall j \in V, \forall t \in T \\
z_{i j t} \geq 0 & \forall i \in N, \forall j \in V, \forall t \in T \\
\sum_{j \in V} z_{i j t} \phi_{i j}=1 & \forall i \in N, \forall t \in T
\end{array}
$$

in which $M$ is a sufficiently large positive number.

Now, the linearized mathematical model can be rewritten as follows.

$$
\begin{array}{lr}
\min _{j \in V} \sum_{t \in T} f_{j t}\left(x_{j t}-x_{j(t-1)}\right)+\sum_{j \in V} \sum_{t \in T} o_{j t} x_{j t} & \\
\sum_{i \in N} \Phi_{i t} p_{i t} \geq \pi_{t} & \forall t \in T \\
\Phi_{i t} \leq 1 & \forall i \in N, \forall t \in T \\
\Phi_{i t} \leq \sum_{j \in V} \phi_{i j} x_{j t} & \forall i \in N, \forall t \in T \\
\sum_{i \in N} p_{i t} \phi_{i j} z_{i j t} \leq \bar{\lambda} x_{j t} & \forall j \in V, \forall t \in T \\
x_{j t} \leq x_{j(t+1)} & \forall j \in V, t \in T \backslash\left\{T_{\max }\right\} \\
\sum_{j \in V} z_{i j t} \phi_{i j}=1 & \forall i \in N, \forall t \in T \\
z_{i j t} \leq M x_{j t} & \forall i \in N, \forall j \in V, \forall t \in T \\
z_{i j t} \leq w_{i t} & \forall i \in N, \forall j \in V, \forall t \in T \\
w_{i t}-z_{i j t} \leq M\left(1-x_{j t}\right) & \forall i \in N, \forall j \in V, \forall t \in T \\
z_{i j t} \geq 0 & \forall i \in N, \forall j \in V, \forall t \in T \\
x_{j t} \in\{0,1\} & \forall j \in V, \forall t \in T
\end{array}
$$

IC-PHNDP belongs to the class of NP-hard problems since its relaxation makes it an uncapaciated facility location problem which has been proven to be an NP-hard problem. Therefore, owing to its combinatorial optimization nature, we propose an efficient VNS to solve it.

\section{Solution procedure}

Variable Neighbourhood Search (Mladenović and Hansen [64]) is a local search procedure based on systematically improving the incumbent solution by applying a set of neighbourhood search structures. VNS has been a popular heuristic in a variety of problems from scheduling (Karimi et al. [65]) and vehicle routing (Belhaiza et al. [66]) to facility location (Davari et al. [67]). Interested readers can refer to Hansen et al. [68] and references therein for further applications of VNS.

Similar to other heuristics, one of the main issues in designing a VNS is to keep a balance between the intensification and diversification of the algorithm. Since its introduction, different scholars have proposed 
various mechanisms to improve this balance. In this paper, we will apply a general skewed version of VNS as given in Algorithm (1).

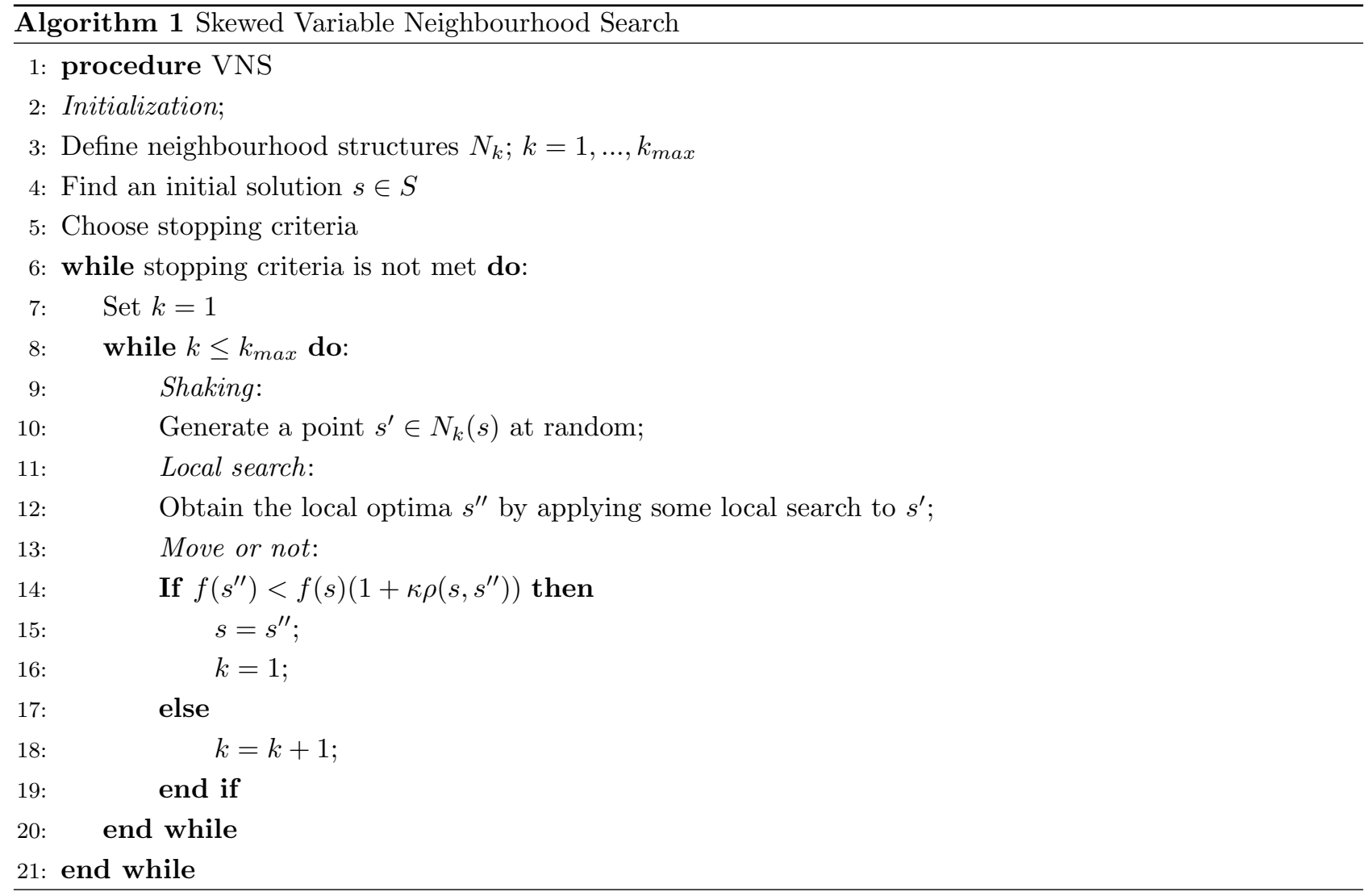

For the distance function $(\rho)$, we propose a function to find the dissimilarity between $s$ and $\bar{s}$. Considering $\theta_{i t}$ as a binary variable taking a value of 1 if a facility at node $i$ is opened at time $t$ and 0 otherwise (the same for $\bar{s}$ ), Equation (31) finds the distance between the two solutions $s$ and $\bar{s}$.

$$
\rho(s, \bar{s})=\frac{\sum_{i=1}^{N} \sum_{t=1}^{T}\left|\theta_{i t}-\bar{\theta}_{i t}\right|}{|N||T|}
$$

In order to increase the diversification ability of the proposed procedure, we allow infeasible solutions to be explored as well. There are two types of infeasibilities in our problem as the violation of the coverage (Constraint (20)) and violation of the congestion (Constraint (23)). We add two penalty terms to the objective function as $\varphi$ and $\psi$ for each unit of violation for the two constraints respectively. These two parameters are updated dynamically during the run to have an optimal trade-off between the intensification and diversification of the procedure. Algorithm (2) presents the procedure to update these parameters. 


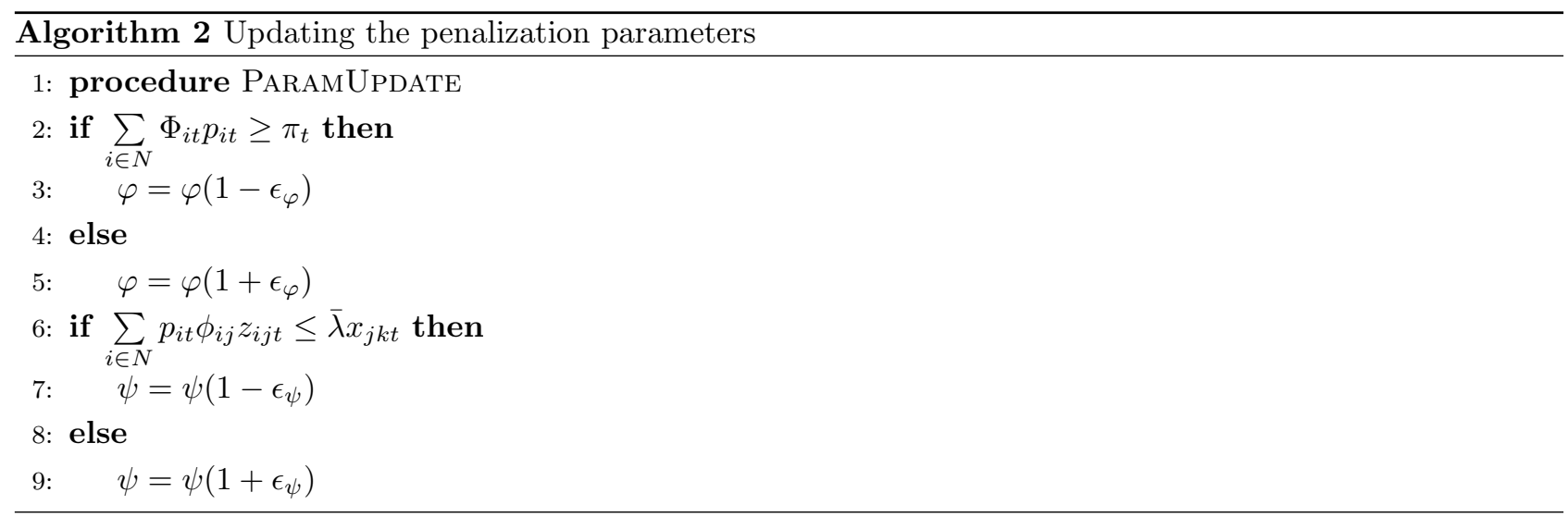

In this section, we will elaborate the encoding scheme, initialisation procedure, and neighbourhood search structures.

\subsection{Solution Representation}

Solution representation plays a crucial role in success of any heuristic method and VNS is not an exception. In this paper, we have used a vector to represent a solution. Assuming $n$ potential facilities to locate and $T_{\max }$ as the number of periods, each vector is composed of $n$ elements each with a value in the range of $\left[0, T_{\max }\right]$ showing the index of the period the facility starts to operate. Since the problem is studied in an uninterrupted settings, this compact representation can be easily transferred to a vector/matrix showing the location of each facility at each time period. The sample solution in Figure (2) shows a problem with eight facilities where a facility is located at node two in the second period and another facility is established at node four in the first period.

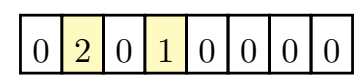

Figure 2: Solution representation

This representation facilitates carrying out neighbourhood search structures quickly which leads to a fast and efficient heuristic. Moreover, the sparse nature of the vector enables the algorithm to benefit from massive memory savings. Hence, we believe that this solution representation is efficient.

\subsection{Initial solution construction}

We employed a fuzzy $c$-means procedure to generate initial solutions which are feasible (interested readers can refer to Sato and Jain [69] for further information about fuzzy $c$-means algorithm and its variants). A sketch of this procedure is given in Algorithm (3). 


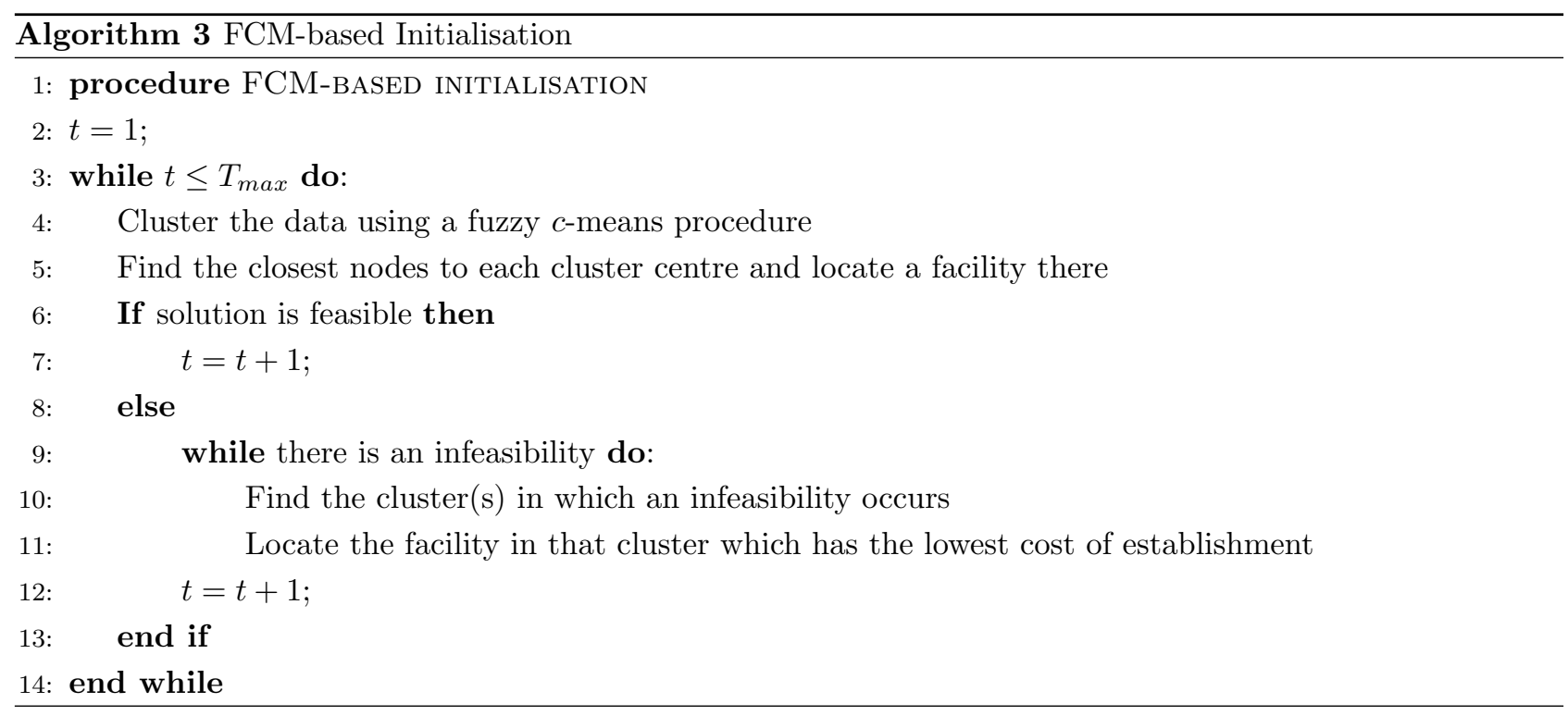

\subsection{Neighbourhood Operators}

We defined a set of five different neighbourhood structures to guide the search and to maintain a balance between intensification and diversification as $\mathcal{N}=\left\{\mathcal{N}_{1}, \mathcal{N}_{2}, \mathcal{N}_{3}, \mathcal{N}_{4}, \mathcal{N}_{5}\right\}$. The moves $\mathcal{N}_{3}-\mathcal{N}_{5}$ have a nested relation as $\mathcal{N}_{3} \subseteq \mathcal{N}_{4} \subseteq \mathcal{N}_{5}$. The following sections explain each structure using an example.

\subsubsection{Backward Shift $\left(\mathcal{N}_{1}\right)$}

The neighbourhood structure shifts back the establishment period of an existing facility. Figure (3) depicts a sample solution where the establishment of facility (2) has been rescheduled to period (1). Please note that this neighbourhood structure can improve the solution by closing a facility or by shifting its establishment period to an earlier one and also make a solution feasible by providing higher service levels for the earlier periods. This operator is invoked repeatedly for all the possible values of $t \in T$ in order to find a better solution.

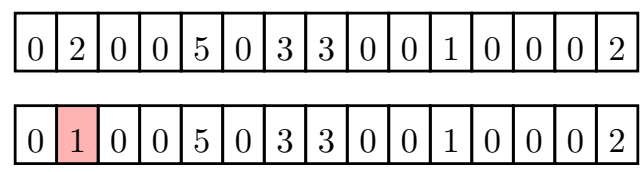

Figure 3: Pushing Back

\subsubsection{Forward Shift $\left(\mathcal{N}_{2}\right)$}

The neighbourhood structure performs in an opposite way to $\mathcal{N}_{1}$ by shifting forward the establishment period of an existing facility. The move is applicable for non-existing facilities as well by locating them in a consequent period. Figure (4) shows a sample solution where the establishment period of facility (7) has been rescheduled to period (4) from period (3). This operator is used repeatedly for all the possible values and the best one is opted for.

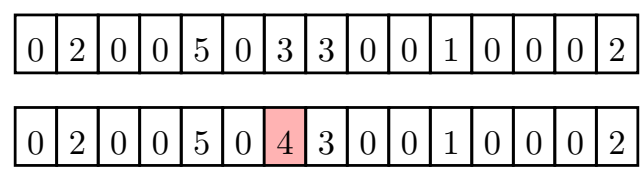

Figure 4: Pulling Forward 


\subsubsection{Swap $\left(\mathcal{N}_{3}-\mathcal{N}_{5}\right)$}

A neighbour of a solution $s$ is obtained by exchanging the values of $q$ pairs of it where $1 \leq q \leq 3$. In other words, the values of a set of $q$ bits are exchanged with the values of a different set of $q$ bits in the solution. Figure (5) presents a sample solution obtained using $q=2$ where the values of two pairs of bits are exchanged (second bit with the eleventh and the sixth bit with the eighth). Our preliminary analysis showed that using the values of more than three for $q$ perturbs the solution to a level that might negatively affect the quality of solutions.

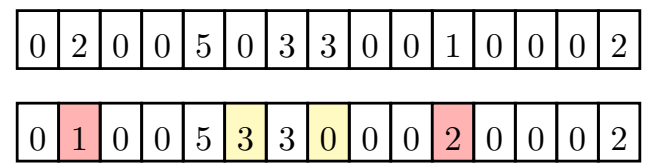

Figure 5: $\operatorname{Swap}(2)$

We will use operators $\mathcal{N}_{3}-\mathcal{N}_{5}$ for the shaking phase and the structures $\mathcal{N}_{1}-\mathcal{N}_{2}$ as the local search procedures. The shaking structures are able to perturb the local optima strongly to increase the diversification of the algorithm and the local search procedures are intensification operators exploring the neighbourhood of a solution to find a better one by slightly changing it.

\subsubsection{Stopping Criteria}

Our experiments showed that for small-scale problems $(|N| \leq 25)$, running the procedure for more than 60 seconds rarely brings about improvement in the solution quality. Hence, the proposed procedure stops after running for 60 seconds regardless of the problem size and its parameters. However, for large-scale problems, we adopted a different stopping criterion based on the number of nodes $(|N|)$ and time periods $(|T|)$ as stopping after $60 \frac{|N||T|}{50}$ seconds. This time corresponds to the wall-clock time of the algorithm including the pre-processing tasks and the reporting time. It should be noted that these stopping criteria are dependent on the hardware specification and the programming language.

\section{$5 \quad$ Numerical experiments}

Since no benchmark instances exists in the literature for IC-PHNDP, we generated a hypothetical set of 216 test problems with different settings ${ }^{1}$. In particular, we considered three dimensions of $|N| \in\{15,20,25\}$. We assumed that there is a direct link between any two nodes enabling the demand to access services in the shortest time.The other settings are shown in Table (2). It should be noted that the third option of $\bar{\lambda}$ in Table (2) is basically the uncapcitated version of the problem where there is no congestion in facilities. For the node distributions, we used the Beta distribution with different $(\alpha, \beta)$ values to have symmetric/non-symmetric and also dense/sparse distributions. Figure (6) depicts the four types of node distributions on the plane with different values of $(\alpha, \beta)$. For the sake of reading the table easier, the total population over the periods is denoted as $\Delta$ as is shown in Equation (32). Finally, the $\pi$ vector was generated using two different parameters imposing different rates of constraint on the problem. In order to address the test problems throughout the paper, we adopt the $|N| /|T| /(\alpha, \beta) / \bar{\lambda} / \pi$ notation.

$$
\Delta=\sum_{i \in N} \sum_{t \in T} p_{i t}
$$

\footnotetext{
${ }^{1}$ Test problems can be shared upon request via email.
} 
Table 2: Parameter settings for the test problems

\begin{tabular}{lllll}
\hline Parameter & Levels & & & \\
\hline$|N|$ & 15 & 20 & 25 & \\
$|T|$ & 1 & 3 & 6 & \\
$(\alpha, \beta)$ & $(1,1)$ & $(2,5)$ & $(5,5)$ & $(1,5)$ \\
$\bar{\lambda}$ & $\frac{2 \Delta}{|N||T|}$ & $\frac{5 \Delta}{|N||T|}$ & $\infty$ & \\
$\pi_{t}$ & $(1-0.7)^{t}$ & $(1-0.95)^{t}$ & & \\
\hline
\end{tabular}

$\operatorname{Beta}(1,1)$
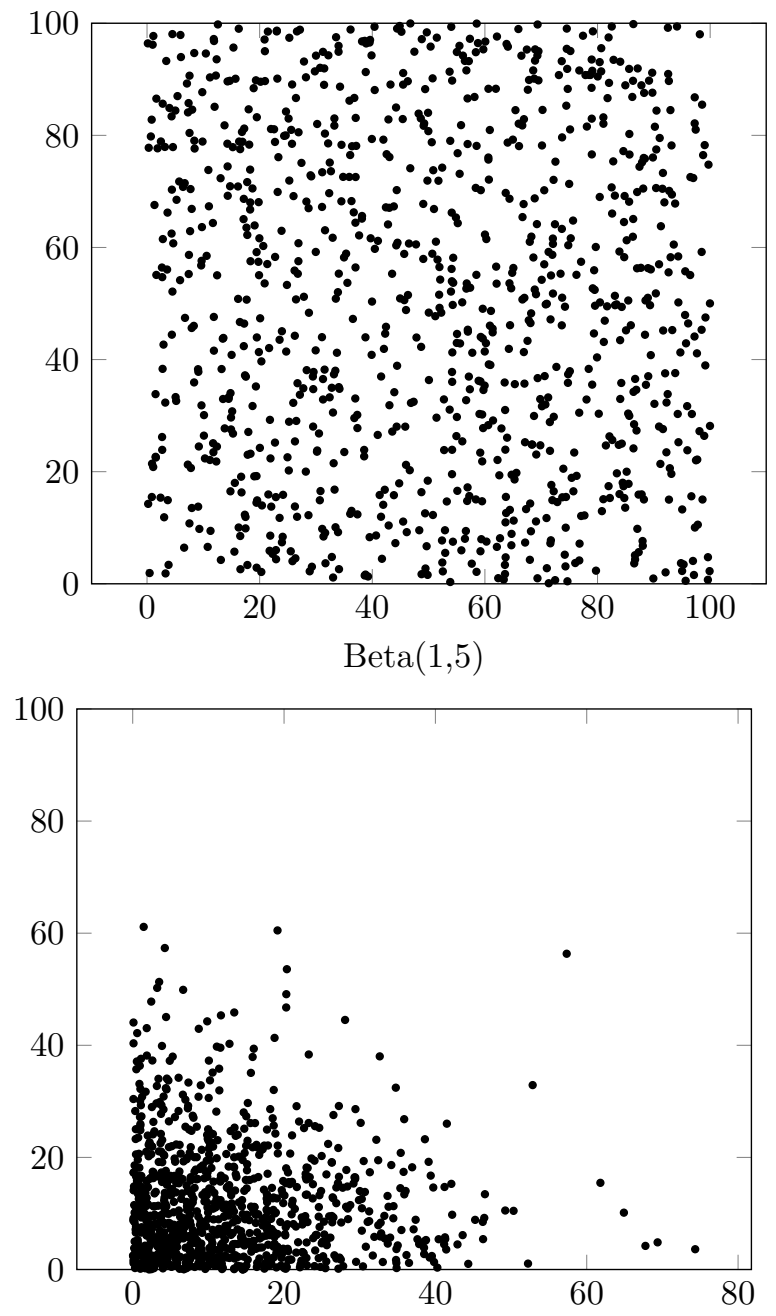

$\operatorname{Beta}(2,5)$
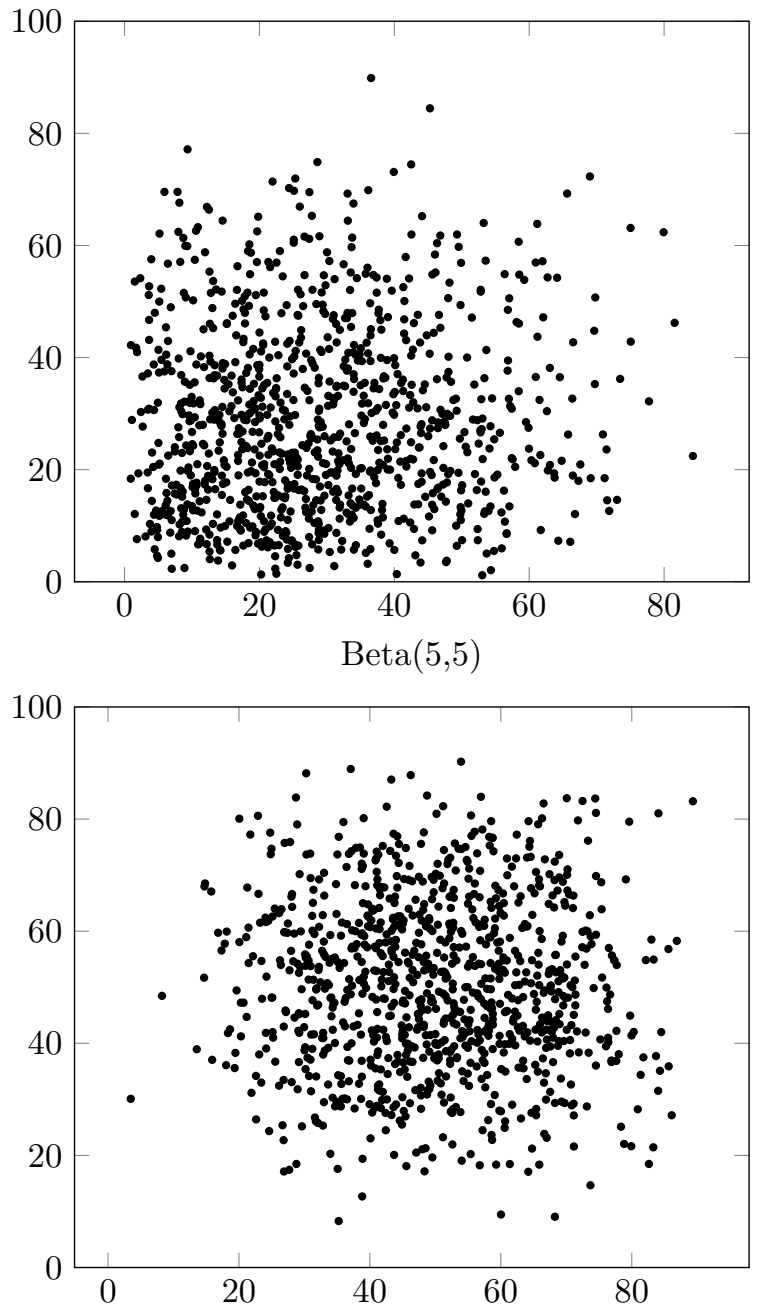

Figure 6: Four demand profiles

All experiments described in this section have been carried out on a laptop with Intel i5 $2.30 \mathrm{GHz} \mathrm{CPU}$ and 16 gigabyte RAM memory. The proposed VNS algorithm was coded in C++ on Visual Studio 2015 and the Gurobi Optimizer was used to solve the MILP model. We allowed the optimizer to run for at most five hours regardless of the problem size. For those instances that Gurobi failed to find the optimal fitness, the lower bound has been reported for the sake of comparison with the proposed VNS. Moreover, we ran our heuristic ten times on each test problem and reported the worst, average, and best performances in terms of the gap to the best found solution. 


\subsection{Parameter tuning}

There are four parameters to be optimised before running the proposed heuristic: the time limit, the maximum neighbourhood size $\left(k_{\max }\right)$, and the values of $\epsilon_{\varphi}$ and $\epsilon_{\psi}$. We found that running the proposed VNS for more than 60 seconds is rarely effective in improving the solution (marginal improvements in seven out of 500 random instances). Hence, we opted for a termination criterion of reaching 60 seconds. Needless to say, running the heuristic for a longer time still improves the solution, but the pace of improvements slows down. It should be mentioned that plotting the solutions shows that the heuristic does not need the whole 60 seconds to reach an optimal solution for the small-scale problems.

The value of $k_{\max }$ has been already tuned as explained earlier to be five. This can be attributed to the fact that for the values of $k$ above five, there is occasionally an improvement seen and the search becomes almost random, negatively affecting the structure of current solution.

In order to find the optimal value of $\epsilon_{\varphi}$ and $\epsilon_{\psi}$, we ran the algorithm ten times with values in the range of [0-0.1] with increments of 0.01 to solve 36 test problems with different number of nodes, number of periods, and distribution of demand nodes and reported the average gaps to the optimal solution. The results for $\epsilon_{\psi}$ were inconclusive and its value has been set to zero. However, as Figure (7) shows, a value of 0.04 for the $\epsilon_{\varphi}$ leads to the best results in terms of the error.

In order to test the superiority of using $\epsilon_{\varphi}=0.04$ over the other values, we performed a Wilcoxin signed-rank test on top of the visual test which showed that the results obtained using $\epsilon_{\varphi}=0.04$ leads to better results compared to the other values.

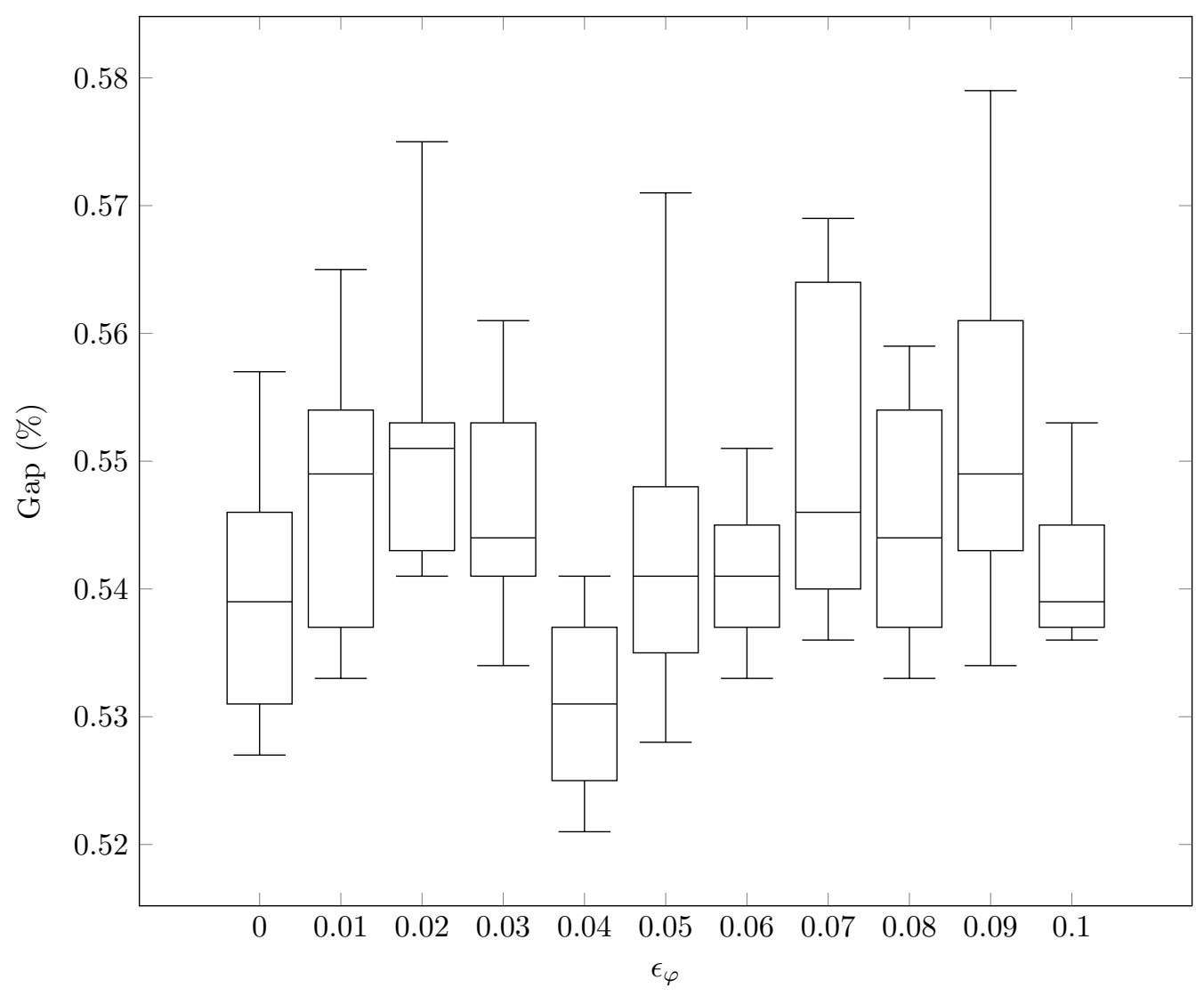

Figure 7: Box-plot of errors using eleven values of $\epsilon_{\varphi}$ 


\section{Results and discussion}

Each of the 216 instances were solved to optimality using Gurobi Optimizer Version 6.5.1 and also ten times using the proposed algorithm. Table (3) summarise the obtained results for both the Gurobi and heuristic runs. The table contains the results for the average performance of the proposed heuristic compared to the Gurobi outputs. While the first four columns represent the problem parameters, the last four columns report on the average gap of the proposed heuristic for each of the demand distribution types.

A detailed summary of the results for each distribution can be found in Tables (4)-(7) in the appendix. While the first six columns of these tables show the problem parameters, the two columns under "Gurobi" give the optimal solution and runtime for each instance respectively whereas the three columns under "Heuristic" give the best, average, and worst results found of the ten runs of the heuristic. Finally, the last three columns report the gaps found in the ten runs of the heuristic. We did not report the runtime needed for heuristics as they were set as 60 seconds for all the instances regardless of the problem settings. Please note that for those instances Gurobi was unable to reach an optimal solution in five hours, the lower bound has been reported and the corresponding row has been made boldfaced. Besides, the rows of those instances for which the heuristic reached the optimal solution at least once have been highlighted. 
Table 3: Summary of the heuristic performance

\begin{tabular}{|c|c|c|c|c|c|c|c|}
\hline \multicolumn{4}{|c|}{ Parameters } & \multicolumn{4}{|c|}{ Average Gap } \\
\hline$|N|$ & $|T|$ & $\lambda$ & $\pi$ & $(1,1)$ & $(1,5)$ & $(2,5)$ & $(5,5)$ \\
\hline 15 & 1 & 2 & 0.7 & $0.00 \%$ & $0.00 \%$ & $0.16 \%$ & $0.15 \%$ \\
\hline 15 & 1 & 2 & 0.95 & $0.00 \%$ & $0.00 \%$ & $0.11 \%$ & $0.21 \%$ \\
\hline 15 & 1 & 5 & 0.7 & $0.00 \%$ & $0.00 \%$ & $0.17 \%$ & $0.16 \%$ \\
\hline 15 & 1 & 5 & 0.95 & $0.00 \%$ & $0.00 \%$ & $0.21 \%$ & $0.29 \%$ \\
\hline 15 & 1 & $\infty$ & 0.7 & $0.00 \%$ & $0.00 \%$ & $0.32 \%$ & $0.18 \%$ \\
\hline 15 & 1 & $\infty$ & 0.95 & $0.00 \%$ & $0.00 \%$ & $0.16 \%$ & $0.15 \%$ \\
\hline 15 & 3 & 2 & 0.7 & $0.82 \%$ & $0.75 \%$ & $0.52 \%$ & $0.24 \%$ \\
\hline 15 & 3 & 2 & 0.95 & $0.58 \%$ & $0.35 \%$ & $0.35 \%$ & $0.82 \%$ \\
\hline 15 & 3 & 5 & 0.7 & $0.51 \%$ & $0.83 \%$ & $0.64 \%$ & $0.35 \%$ \\
\hline 15 & 3 & 5 & 0.95 & $0.56 \%$ & $0.58 \%$ & $0.30 \%$ & $0.94 \%$ \\
\hline 15 & 3 & $\infty$ & 0.7 & $0.45 \%$ & $0.36 \%$ & $0.46 \%$ & $0.17 \%$ \\
\hline 15 & 3 & $\infty$ & 0.95 & $0.31 \%$ & $0.29 \%$ & $0.55 \%$ & $0.24 \%$ \\
\hline 15 & 6 & 2 & 0.7 & $0.53 \%$ & $0.91 \%$ & $1.13 \%$ & $0.95 \%$ \\
\hline 15 & 6 & 2 & 0.95 & $1.07 \%$ & $0.65 \%$ & $0.96 \%$ & $1.33 \%$ \\
\hline 15 & 6 & 5 & 0.7 & $0.51 \%$ & $0.85 \%$ & $0.86 \%$ & $1.22 \%$ \\
\hline 15 & 6 & 5 & 0.95 & $0.59 \%$ & $0.39 \%$ & $1.14 \%$ & $0.40 \%$ \\
\hline 15 & 6 & $\infty$ & 0.7 & $0.07 \%$ & $0.35 \%$ & $0.33 \%$ & $0.38 \%$ \\
\hline 15 & 6 & $\infty$ & 0.95 & $0.35 \%$ & $0.08 \%$ & $0.63 \%$ & $0.40 \%$ \\
\hline 20 & 1 & 2 & 0.7 & $0.12 \%$ & $0.13 \%$ & $0.26 \%$ & $0.27 \%$ \\
\hline 20 & 1 & 2 & 0.95 & $0.21 \%$ & $0.36 \%$ & $0.22 \%$ & $0.47 \%$ \\
\hline 20 & 1 & 5 & 0.7 & $0.04 \%$ & $0.29 \%$ & $0.34 \%$ & $0.51 \%$ \\
\hline 20 & 1 & 5 & 0.95 & $0.12 \%$ & $0.19 \%$ & $0.12 \%$ & $0.41 \%$ \\
\hline 20 & 1 & $\infty$ & 0.7 & $0.00 \%$ & $0.09 \%$ & $0.18 \%$ & $0.22 \%$ \\
\hline 20 & 1 & $\infty$ & 0.95 & $0.00 \%$ & $0.16 \%$ & $0.09 \%$ & $0.14 \%$ \\
\hline 20 & 3 & 2 & 0.7 & $0.88 \%$ & $0.84 \%$ & $1.33 \%$ & $1.12 \%$ \\
\hline 20 & 3 & 2 & 0.95 & $1.15 \%$ & $1.08 \%$ & $0.88 \%$ & $1.36 \%$ \\
\hline 20 & 3 & 5 & 0.7 & $0.85 \%$ & $1.08 \%$ & $0.97 \%$ & $1.58 \%$ \\
\hline 20 & 3 & 5 & 0.95 & $0.81 \%$ & $0.96 \%$ & $0.72 \%$ & $1.44 \%$ \\
\hline 20 & 3 & $\infty$ & 0.7 & $0.74 \%$ & $0.77 \%$ & $0.77 \%$ & $1.43 \%$ \\
\hline 20 & 3 & $\infty$ & 0.95 & $0.56 \%$ & $0.76 \%$ & $0.29 \%$ & $0.94 \%$ \\
\hline 20 & 6 & 2 & 0.7 & $1.05 \%$ & $1.39 \%$ & $1.62 \%$ & $1.39 \%$ \\
\hline 20 & 6 & 2 & 0.95 & $1.06 \%$ & $1.20 \%$ & $1.32 \%$ & $1.29 \%$ \\
\hline 20 & 6 & 5 & 0.7 & $0.99 \%$ & $0.95 \%$ & $1.18 \%$ & $1.47 \%$ \\
\hline 20 & 6 & 5 & 0.95 & $1.32 \%$ & $1.18 \%$ & $1.13 \%$ & $1.33 \%$ \\
\hline 20 & 6 & $\infty$ & 0.7 & $0.78 \%$ & $0.86 \%$ & $1.02 \%$ & $1.34 \%$ \\
\hline 20 & 6 & $\infty$ & 0.95 & $0.32 \%$ & $0.40 \%$ & $0.90 \%$ & $0.96 \%$ \\
\hline 25 & 1 & 2 & 0.7 & $0.37 \%$ & $0.42 \%$ & $1.01 \%$ & $0.74 \%$ \\
\hline 25 & 1 & 2 & 0.95 & $0.47 \%$ & $0.45 \%$ & $0.85 \%$ & $0.46 \%$ \\
\hline 25 & 1 & 5 & 0.7 & $0.49 \%$ & $0.44 \%$ & $0.44 \%$ & $0.64 \%$ \\
\hline 25 & 1 & 5 & 0.95 & $0.05 \%$ & $0.51 \%$ & $0.69 \%$ & $0.66 \%$ \\
\hline 25 & 1 & $\infty$ & 0.7 & $0.12 \%$ & $0.62 \%$ & $0.82 \%$ & $0.35 \%$ \\
\hline 25 & 1 & $\infty$ & 0.95 & $0.05 \%$ & $0.49 \%$ & $0.42 \%$ & $0.34 \%$ \\
\hline 25 & 3 & 2 & 0.7 & $1.77 \%$ & $0.73 \%$ & $1.07 \%$ & $1.63 \%$ \\
\hline 25 & 3 & 2 & 0.95 & $1.23 \%$ & $0.89 \%$ & $1.18 \%$ & $1.51 \%$ \\
\hline 25 & 3 & 5 & 0.7 & $1.12 \%$ & $1.08 \%$ & $1.04 \%$ & $1.68 \%$ \\
\hline 25 & 3 & 5 & 0.95 & $1.76 \%$ & $0.70 \%$ & $1.47 \%$ & $2.26 \%$ \\
\hline 25 & 3 & $\infty$ & 0.7 & $0.85 \%$ & $0.53 \%$ & $0.91 \%$ & $1.76 \%$ \\
\hline 25 & 3 & $\infty$ & 0.95 & $1.25 \%$ & $0.38 \%$ & $0.77 \%$ & $1.64 \%$ \\
\hline 25 & 6 & 2 & 0.7 & $2.31 \%$ & $1.65 \%$ & $1.56 \%$ & $1.79 \%$ \\
\hline 25 & 6 & 2 & 0.95 & $4.25 \%$ & $2.03 \%$ & $1.08 \%$ & $1.21 \%$ \\
\hline 25 & 6 & 5 & 0.7 & $2.32 \%$ & $1.22 \%$ & $2.40 \%$ & $1.26 \%$ \\
\hline 25 & 6 & 5 & 0.95 & $1.67 \%$ & $1.26 \%$ & $1.29 \%$ & $1.50 \%$ \\
\hline 25 & 6 & $\infty$ & 0.7 & $1.20 \%$ & $0.94 \%$ & $1.11 \%$ & $1.41 \%$ \\
\hline 25 & 0 & $\infty$ & 0.95 & $1.52 \%$ & $0.84 \%$ & $0.85 \%$ & $0.92 \%$ \\
\hline
\end{tabular}


Results show that while for the instances with 15 nodes, Gurobi was able to solve all the instances to optimality within 11 minutes, it needed more than two hours to solve some instances with $|N|=20$, and failed to reach an optimal solution for some of the instances with $|N|=25$ within five hours. Our experiments showed that for larger node sizes, Gurobi failed to reach an optimal solution in much longer times. However, the proposed heuristic consumed considerably lower times (60 seconds) to reach solutions which are not worse than $0.55 \%$ from the optimal solution on average. In particular, for the large-scale problems, the proposed heuristic reached solutions which are $0.81 \%$ higher than the optimal solutions on average. However, as Figure (8) shows, for a case with 25 nodes and six periods, Gurobi was unable to reach an optimal solution with less than $15 \%$ gap to the optimal within two hours. It should be emphasised that there is no guarantee with the proposed heuristic to reach an optimal solution. However, for larger instances in which Gurobi is unable to find the optimal solution, the heuristic can offer a near-optimal solution as shown in the numerical experiments.

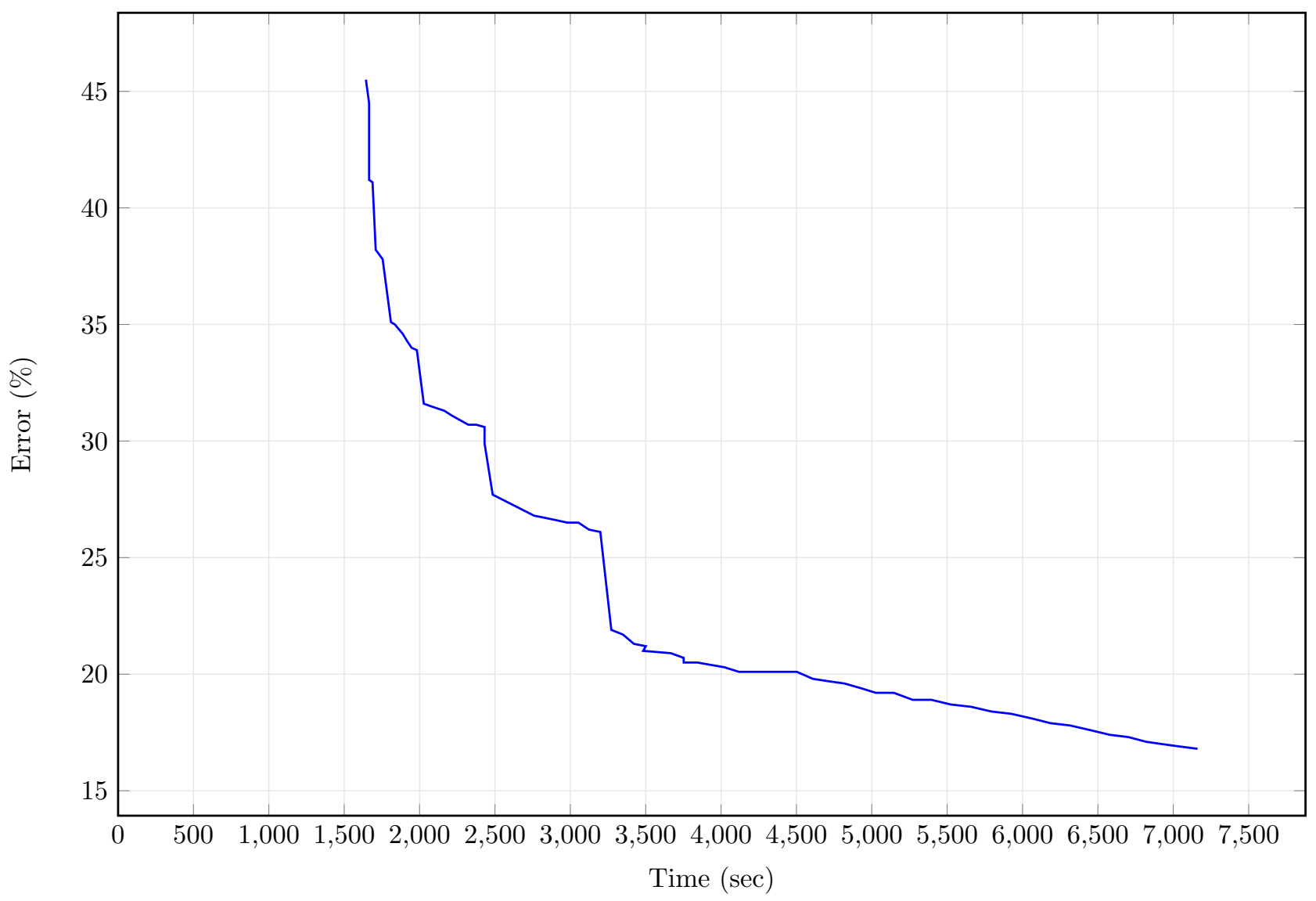

Figure 8: Performance of Gurobi on an instance of 25/6/(1,5)/2/0.7

One of the observations is that the computational requirement grows exponentially by an increase in the number of nodes and periods, whereas an increase in the value of congestion parameter $(\bar{\lambda})$ is strongly correlated with a decrease in the runtime. While all the instances with a $\lambda=\infty$ were solved in less than a minute, only 24 out of 48 with $\lambda=2$ or $\lambda=5$ and $|N|=25$ were solved optimally in less than ten minutes. Moreover, the heuristic reached the optimal solution in 36 and resulted in solutions within an error margin of one percent in 186 out of 216 instances which are clear indications that the proposed heuristic performs significantly better in terms of the runtime while its solution quality is comparable to Gurobi.

Another observation in the running of the heuristic was the ability of the proposed heuristic to avoid getting stuck in local optima. For instance, Figure (9) depicts a sample run of the heuristic for 60 seconds where the algorithm reduced the gap gradually without being stuck in local optima. 


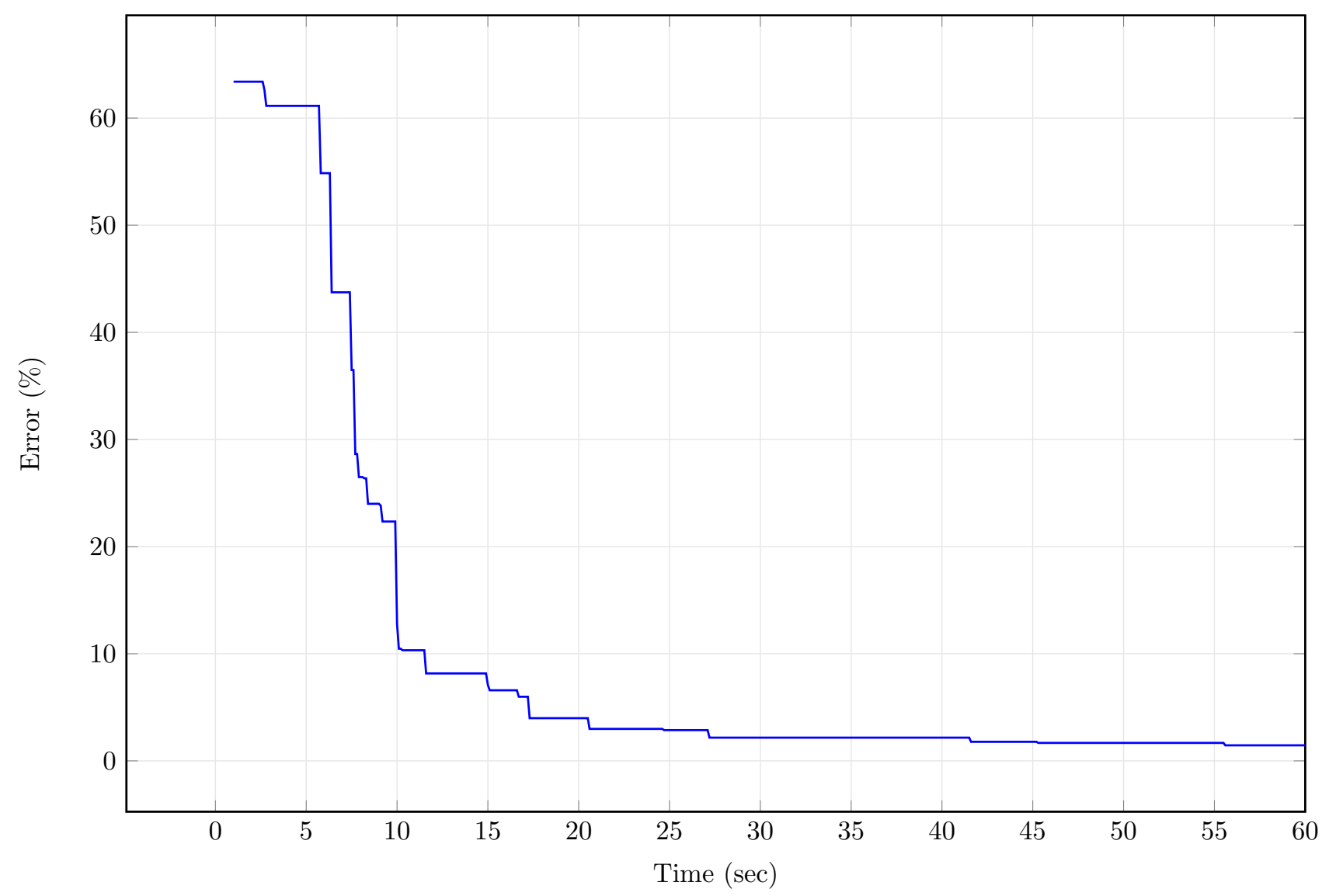

Figure 9: Performance of the algorithm on an 25/6/(1,1)/5/0.7

In order to analyse the efficiency of the proposed heuristic, we used two measures simultaneously, namely the runtime and also the Relative Percentage Deviation (RPD) as Equation (33):

$$
R P D=\frac{z-z^{*}}{z^{*}}
$$

where $z$ and $z^{*}$ are the best found fitness using the proposed VNS and the optimal value found using Gurobi respectively. While for small-sized problems, we compared the performance of the proposed procedure with the optimal solution found with Gurobi, the performance for the larger ones, for which Gurobi was unable to find the optimal solution within five hours, was compared with the lower bounds.

We observe that the performance of the proposed heuristic is not far from the Gurobi results with an average error of $0.81 \%$ for those instances Gurobi managed to solve. However, in terms of the runtime, VNS is better by an order of magnitude. The gap of VNS is higher for those instances with a lower value of $\bar{\lambda}$ which can be attributed to the fact that these instances are more difficult to solve. However, this issue is offset considering the fact that the time needed to solve these instances to optimality is high as Gurobi fails to reach an optimal even in five hours (For some instances, no optimal solution is found within even twelve hours). Furthermore, in $50 \%$ of test instances, the proposed general SVNS could reach the optimal solution in at least one of the runs and in almost $20 \%$ of the cases, it gets the optimal results in all the ten runs. Therefore, the proposed algorithm is capable in reaching high quality solutions in considerably less time than Gurobi.

In order to examine the performance of the proposed heuristic for larger datasets, we have carried out a set of complimentary analysis for larger datasets of $|N| \in\{100,1000\}$ with increments of 100 and the results are given in Tables (8)-(10). Since Gurobi fails to find the optimal solutions for these instances in a reasonable time (even a week), we were unable to compare the performance of heuristic with the optimal results. However, the best, average, and worst solutions of the ten runs are reported along with the standard deviation of the 
results in the last column which has been shown as $\sigma$.

We have done a set of additional tests to find out if the performance of the heuristic is affected by using incomplete graphs. To this end, we used the standard $p$-median test problems of Beasley [70] for $|N| \in$ $\{100,200,300,400,500\},|T| \in\{1,3,6\}, \bar{\lambda}=50$ and $\pi=0.7$. Results of these test problems are given in Table (11) showing that the heuristic performs well for the incomplete graphs as well, leading to consistent results.

We carried out an additional test to examine the efficiency of the skewed VNS and if it makes a significant difference compared to the general VNS. To do so, we ran the same test problems with a general VNS $(\kappa=0)$ and compared the means using the unpaired $t$-test. These analyses have led to $p$-values equal to $0.093,0.066$, 0.041 , and 0.085 for $(\alpha, \beta)=(1,1),(\alpha, \beta)=(1,5),(\alpha, \beta)=(2,5)$, and $(\alpha, \beta)=(5,5)$ respectively. Therefore, we can conclude that there is a significant difference between the general VNS and the proposed skewed VNS at ten percent level of confidence.

\section{Conclusion and future research}

The literature of facility location models has relatively less publications for multi-period models than singleperiod ones which can be attributed to the complexity of these problems and the high computational effort needed to solve them. Moreover, the cooperative facility location problem is relatively new. In this paper, we tried to fill this gap by considering a multi-period cooperative facility location problem for preventive health care centres. We proposed a linear integer programming model and developed an efficient heuristic to solve it. Our experiments using a set of randomly generated data showed that the proposed heuristic is able to reach near-optimal solutions in considerably less runtimes compared to the optimal solutions found by Gurobi Optimizer.

We believe that future research stems from considering probabilistic choice environment, assuming uncertain travel times or developing other heuristics. Another appealing future research is to assume a case in which preventive facilities are dynamic, like immunisation programs. Then, the problem is to locate facilities dynamically and to decide on the time the locations should be changed. As another possible extension to the IC-PHNDP, other qualitative factors such as quality of the healthcare facility, availability of amenities near the facility, etc. which also influence the attractiveness of the healthcare facility besides the proximity, can also be incorporated while modelling participation to preventive programs. Another interesting area of research would be to compare the performance of Gurobi with other solvers to find out if any solver shows a better performance to solve IC-PHNDP. Last but not least, one can model the participation by means of fuzzy numbers rather than crisp numbers and to utilise fuzzy mathematical programming approaches. 


\section{Appendix. Numerical Results}

Table 4: Results for the instance with $(\alpha, \beta)=(1,1)$

\begin{tabular}{|c|c|c|c|c|c|c|c|c|c|c|c|c|c|}
\hline \multirow{2}{*}{$|N|$} & \multirow{2}{*}{$|T|$} & \multirow{2}{*}{$\alpha$} & \multirow{2}{*}{$\beta$} & \multirow{2}{*}{$\bar{\lambda}$} & \multirow{2}{*}{$\pi$} & \multicolumn{2}{|l|}{ Gurobi } & \multicolumn{3}{|l|}{ Heuristic } & \multicolumn{3}{|l|}{ Gap } \\
\hline & & & & & & Objective & Time $(\mathrm{s})$ & Best & Average & Worst & Best & Average & Worst \\
\hline 15 & 1 & 1 & 1 & 2 & 0.7 & 334,687 & 2.0 & 334,687 & 334,687 & 334,687 & $0.00 \%$ & $0.00 \%$ & $0.00 \%$ \\
\hline 15 & 1 & 1 & 1 & 2 & 0.95 & 334,687 & 2.1 & 334,687 & 334,687 & 334,687 & $0.00 \%$ & $0.00 \%$ & $0.00 \%$ \\
\hline 15 & 1 & 1 & 1 & 5 & 0.7 & 86,914 & 1 & 86,914 & 86,914 & 86,914 & $0.00 \%$ & $0.00 \%$ & $0.00 \%$ \\
\hline 15 & 1 & 1 & 1 & 5 & 0.95 & 86,914 & 1.1 & 86,914 & 86,914 & 86,914 & $0.00 \%$ & $0.00 \%$ & $0.00 \%$ \\
\hline 15 & 1 & 1 & 1 & $\infty$ & 0.7 & 29,626 & $<1$ & 29,626 & 29,626 & 29,626 & $0.00 \%$ & $0.00 \%$ & $0.00 \%$ \\
\hline 15 & 1 & 1 & 1 & $\infty$ & 0.95 & 29,626 & $<1$ & 29,626 & 29,626 & 29,626 & $0.00 \%$ & $0.00 \%$ & $0.00 \%$ \\
\hline 15 & 3 & 1 & 1 & 2 & 0.7 & 412,718 & $<1$ & 414,823 & 416,086 & 416,423 & $0.51 \%$ & $0.82 \%$ & $0.90 \%$ \\
\hline 15 & 3 & 1 & 1 & 2 & 0.95 & 412,718 & $<1$ & 414,905 & 415,124 & 416,568 & $0.53 \%$ & $0.58 \%$ & $0.93 \%$ \\
\hline 15 & 3 & 1 & 1 & 5 & 0.7 & 141,895 & 4.5 & 142,548 & 142,613 & 142,900 & $0.46 \%$ & $0.51 \%$ & $0.71 \%$ \\
\hline 15 & 3 & 1 & 1 & 5 & 0.95 & 107,187 & 4.2 & 107,734 & 107,788 & 108,330 & $0.51 \%$ & $0.56 \%$ & $1.07 \%$ \\
\hline 15 & 3 & 1 & 1 & $\infty$ & 0.7 & 107,929 & 1.0 & 108,231 & 108,413 & 108,509 & $0.28 \%$ & $0.45 \%$ & $0.54 \%$ \\
\hline 15 & 3 & 1 & 1 & $\infty$ & 0.95 & 44,809 & $<1$ & 44,926 & 44,949 & 45,047 & $0.26 \%$ & $0.31 \%$ & $0.53 \%$ \\
\hline 15 & 6 & 1 & 1 & 2 & 0.7 & 607,902 & 283.1 & 610,394 & 611,142 & 611,790 & $0.41 \%$ & $0.53 \%$ & $0.64 \%$ \\
\hline 15 & 6 & 1 & 1 & 2 & 0.95 & 607,902 & 250.0 & 611,975 & 614,419 & 616,374 & $0.67 \%$ & $1.07 \%$ & $1.39 \%$ \\
\hline 15 & 6 & 1 & 1 & 5 & 0.7 & 263,743 & 6.5 & 264,640 & 265,088 & 265,626 & $0.34 \%$ & $0.51 \%$ & $0.71 \%$ \\
\hline 15 & 6 & 1 & 1 & 5 & 0.95 & 163,405 & 47.0 & 164,042 & 164,361 & 165,221 & $0.39 \%$ & $0.59 \%$ & $1.11 \%$ \\
\hline 15 & 6 & 1 & 1 & $\infty$ & 0.7 & 246,927 & 2.4 & 247,075 & 247,090 & 247,204 & $0.06 \%$ & $0.07 \%$ & $0.11 \%$ \\
\hline 15 & 6 & 1 & 1 & $\infty$ & 0.95 & 79,688 & $<1$ & 79,919 & 79,965 & 80,159 & $0.29 \%$ & $0.35 \%$ & $0.59 \%$ \\
\hline 20 & 1 & 1 & 1 & 2 & 0.7 & 452,865 & 19.9 & 452,865 & 453,408 & 453,898 & $0.00 \%$ & $0.12 \%$ & $0.23 \%$ \\
\hline 20 & 1 & 1 & 1 & 2 & 0.95 & 452,865 & 21.1 & 452,865 & 453,816 & 454,006 & $0.00 \%$ & $0.21 \%$ & $0.25 \%$ \\
\hline 20 & 1 & 1 & 1 & 5 & 0.7 & 107,534 & 1.6 & 107,534 & 107,577 & 107,620 & $0.00 \%$ & $0.04 \%$ & $0.08 \%$ \\
\hline 20 & 1 & 1 & 1 & 5 & 0.95 & 107,534 & 1.5 & 107,534 & 107,663 & 107,740 & $0.00 \%$ & $0.12 \%$ & $0.19 \%$ \\
\hline 20 & 1 & 1 & 1 & $\infty$ & 0.7 & 29,626 & $<1$ & 29,626 & 29,626 & 29,626 & $0.00 \%$ & $0.00 \%$ & $0.00 \%$ \\
\hline 20 & 1 & 1 & 1 & $\infty$ & 0.95 & 29,626 & $<1$ & 29,626 & 29,626 & 29,626 & $0.00 \%$ & $0.00 \%$ & $0.00 \%$ \\
\hline 20 & 3 & 1 & 1 & 2 & 0.7 & 581,278 & 496.4 & 585,521 & 586,370 & 588,916 & $0.73 \%$ & $0.88 \%$ & $1.31 \%$ \\
\hline 20 & 3 & 1 & 1 & 2 & 0.95 & 581,278 & 446.3 & 585,463 & 587,974 & 594,671 & $0.72 \%$ & $1.15 \%$ & $2.30 \%$ \\
\hline 20 & 3 & 1 & 1 & 5 & 0.7 & 165,260 & 17.2 & 166,334 & 166,656 & 167,355 & $0.65 \%$ & $0.85 \%$ & $1.27 \%$ \\
\hline 20 & 3 & 1 & 1 & 5 & 0.95 & 150,619 & 20.8 & 151,432 & 151,839 & 152,937 & $0.54 \%$ & $0.81 \%$ & $1.54 \%$ \\
\hline 20 & 3 & 1 & 1 & $\infty$ & 0.7 & 122,044 & 2.4 & 122,642 & 122,941 & 123,031 & $0.49 \%$ & $0.74 \%$ & $0.81 \%$ \\
\hline 20 & 3 & 1 & 1 & $\infty$ & 0.95 & 44,809 & $<1$ & 44,975 & 45,058 & 45,306 & $0.37 \%$ & $0.56 \%$ & $1.11 \%$ \\
\hline 20 & 6 & 1 & 1 & 2 & 0.7 & 703,727 & 6274.2 & 710,412 & 711,081 & 716,964 & $0.95 \%$ & $1.05 \%$ & $1.88 \%$ \\
\hline 20 & 6 & 1 & 1 & 2 & 0.95 & 737,308 & $1,937.2$ & 743,796 & 745,094 & 752,880 & $0.88 \%$ & $1.06 \%$ & $2.11 \%$ \\
\hline 20 & 6 & 1 & 1 & 5 & 0.7 & 342,645 & 315.6 & 344,769 & 346,044 & 347,744 & $0.62 \%$ & $0.99 \%$ & $1.49 \%$ \\
\hline 20 & 6 & 1 & 1 & 5 & 0.95 & 219,152 & 705.9 & 221,081 & 222,045 & 224,359 & $0.88 \%$ & $1.32 \%$ & $2.38 \%$ \\
\hline 20 & 6 & 1 & 1 & $\infty$ & 0.7 & 298,106 & 7.6 & 300,223 & 300,434 & 301,831 & $0.71 \%$ & $0.78 \%$ & $1.25 \%$ \\
\hline 20 & 6 & 1 & 1 & $\infty$ & 0.95 & 81,201 & 2.1 & 81,436 & 81,460 & 81,719 & $0.29 \%$ & $0.32 \%$ & $0.64 \%$ \\
\hline 25 & 1 & 1 & 1 & 2 & 0.7 & 427,843 & 42.3 & 428,827 & 429,417 & 430,834 & $0.23 \%$ & $0.37 \%$ & $0.70 \%$ \\
\hline 25 & 1 & 1 & 1 & 2 & 0.95 & 427,843 & 41.1 & 429,683 & 429,867 & 431,688 & $0.43 \%$ & $0.47 \%$ & $0.90 \%$ \\
\hline 25 & 1 & 1 & 1 & 5 & 0.7 & 122,433 & 6.8 & 122,862 & 123,033 & 123,153 & $0.35 \%$ & $0.49 \%$ & $0.59 \%$ \\
\hline 25 & 1 & 1 & 1 & 5 & 0.95 & 122,433 & 7.0 & 122,666 & 122,735 & 123,008 & $0.19 \%$ & $0.25 \%$ & $0.47 \%$ \\
\hline 25 & 1 & 1 & 1 & $\infty$ & 0.7 & 29,626 & $<1$ & 29,659 & 29,662 & 29,680 & $0.11 \%$ & $0.12 \%$ & $0.18 \%$ \\
\hline 25 & 1 & 1 & 1 & $\infty$ & 0.95 & 29,626 & $<1$ & 29,626 & 29,641 & 29,647 & $0.00 \%$ & $0.05 \%$ & $0.07 \%$ \\
\hline 25 & 3 & 1 & 1 & 2 & 0.7 & 589,540 & $1,808.7$ & 596,497 & 599,975 & 608,323 & $1.18 \%$ & $1.77 \%$ & $3.19 \%$ \\
\hline 25 & 3 & 1 & 1 & 2 & 0.95 & 589,540 & $2,200.0$ & 594,728 & 596,803 & 598,256 & $0.88 \%$ & $1.23 \%$ & $1.48 \%$ \\
\hline 25 & 3 & 1 & 1 & 5 & 0.7 & 197,395 & 326.0 & 199,408 & 199,610 & 200,496 & $1.02 \%$ & $1.12 \%$ & $1.57 \%$ \\
\hline 25 & 3 & 1 & 1 & 5 & 0.95 & 173,639 & 186.1 & 175,983 & 176,686 & 179,429 & $1.35 \%$ & $1.76 \%$ & $3.33 \%$ \\
\hline 25 & 3 & 1 & 1 & $\infty$ & 0.7 & 127,596 & $<1$ & 128,591 & 128,691 & 128,800 & $0.78 \%$ & $0.86 \%$ & $0.94 \%$ \\
\hline 25 & 3 & 1 & 1 & $\infty$ & 0.95 & 43,970 & $<1$ & 44,335 & 44,517 & 44,846 & $0.83 \%$ & $1.25 \%$ & $1.99 \%$ \\
\hline 25 & 6 & 1 & 1 & 2 & 0.7 & 838,646 & 9472.7 & 851,561 & 858,019 & 867,705 & $1.54 \%$ & $2.31 \%$ & $3.47 \%$ \\
\hline 25 & 6 & 1 & 1 & 2 & 0.95 & 727,461 & 18,000 & 740,046 & 743,821 & 745,457 & $3.73 \%$ & $4.25 \%$ & $4.47 \%$ \\
\hline 25 & 6 & 1 & 1 & 5 & 0.7 & 382,977 & 889.7 & 388,530 & 391,862 & 395,416 & $1.45 \%$ & $2.32 \%$ & $3.25 \%$ \\
\hline 25 & 6 & 1 & 1 & 5 & 0.95 & 246,099 & 2802.2 & 249,520 & 250,204 & 251,025 & $1.39 \%$ & $1.67 \%$ & $2.00 \%$ \\
\hline 25 & 6 & 1 & 1 & $\infty$ & 0.7 & 322,585 & 15.6 & 325,553 & 326,443 & 329,530 & $0.92 \%$ & $1.20 \%$ & $2.15 \%$ \\
\hline 25 & 6 & 1 & 1 & $\infty$ & 0.95 & 79688 & 2.5 & 80,493 & 80,895 & 81,257 & $1.01 \%$ & $1.52 \%$ & $1.97 \%$ \\
\hline
\end{tabular}


Table 5: Results for the instance with $(\alpha, \beta)=(1,5)$

\begin{tabular}{|c|c|c|c|c|c|c|c|c|c|c|c|c|c|}
\hline \multirow{2}{*}{$|N|$} & \multirow{2}{*}{$|T|$} & \multirow{2}{*}{$\alpha$} & \multirow{2}{*}{$\beta$} & \multirow{2}{*}{$\bar{\lambda}$} & \multirow{2}{*}{$\pi$} & \multicolumn{2}{|l|}{ Gurobi } & \multicolumn{3}{|c|}{ Heuristic } & \multicolumn{3}{|l|}{ Gap } \\
\hline & & & & & & Objective & Time $(\mathrm{s})$ & Best & Average & Worst & Best & Average & Worst \\
\hline 15 & 1 & 1 & 5 & 2 & 0.7 & 276,585 & 1.9 & 276,585 & 27,6585 & 276,585 & $0.00 \%$ & $0.00 \%$ & $0.00 \%$ \\
\hline 15 & 1 & 1 & 5 & 2 & 0.95 & 276,585 & 1.9 & 276,585 & 276,585 & 276,585 & $0.00 \%$ & $0.00 \%$ & $0.00 \%$ \\
\hline 15 & 1 & 1 & 5 & 5 & 0.7 & 87,198 & $<1$ & 87,198 & 87,198 & 87,198 & $0.00 \%$ & $0.00 \%$ & $0.00 \%$ \\
\hline 15 & 1 & 1 & 5 & 5 & 0.95 & 87,198 & $<1$ & 87,198 & 87,198 & 87,198 & $0.00 \%$ & $0.00 \%$ & $0.00 \%$ \\
\hline 15 & 1 & 1 & 5 & $\infty$ & 0.7 & 13,661 & $<1$ & 13,661 & 13,661 & 13,661 & $0.00 \%$ & $0.00 \%$ & $0.00 \%$ \\
\hline 15 & 1 & 1 & 5 & $\infty$ & 0.95 & 13,661 & $<1$ & 13,661 & 13,661 & 13,661 & $0.00 \%$ & $0.00 \%$ & $0.00 \%$ \\
\hline 15 & 3 & 1 & 5 & 2 & 0.7 & 368,519 & 72.1 & 370,656 & 371,298 & 371,575 & $0.58 \%$ & $0.75 \%$ & $0.83 \%$ \\
\hline 15 & 3 & 1 & 5 & 2 & 0.95 & 368,519 & 56.1 & 369,588 & 369,801 & 369,930 & $0.29 \%$ & $0.35 \%$ & $0.38 \%$ \\
\hline 15 & 3 & 1 & 5 & 5 & 0.7 & 115592 & 4.2 & 116,228 & 116,546 & 116,927 & $0.55 \%$ & $0.83 \%$ & $1.16 \%$ \\
\hline 15 & 3 & 1 & 5 & 5 & 0.95 & 115592 & 3.6 & 116,008 & 116,258 & 116,591 & $0.36 \%$ & $0.58 \%$ & $0.86 \%$ \\
\hline 15 & 3 & 1 & 5 & $\infty$ & 0.7 & 37,090 & $<1$ & 37,194 & 37,225 & 37,266 & $0.28 \%$ & $0.36 \%$ & $0.47 \%$ \\
\hline 15 & 3 & 1 & 5 & $\infty$ & 0.95 & 22250 & $<1$ & 22,297 & 22,315 & 22,322 & $0.21 \%$ & $0.29 \%$ & $0.32 \%$ \\
\hline 15 & 6 & 1 & 5 & 2 & 0.7 & 449,711 & 189.8 & 452,634 & 453,803 & 454,622 & $0.65 \%$ & $0.91 \%$ & $1.09 \%$ \\
\hline 15 & 6 & 1 & 5 & 2 & 0.95 & 449,711 & 176.3 & 452,364 & 452,630 & 455,548 & $0.59 \%$ & $0.65 \%$ & $1.30 \%$ \\
\hline 15 & 6 & 1 & 5 & 5 & 0.7 & 149,682 & 16.2 & 150,745 & 150,957 & 151,722 & $0.71 \%$ & $0.85 \%$ & $1.36 \%$ \\
\hline 15 & 6 & 1 & 5 & 5 & 0.95 & 143,061 & 10.0 & 143,562 & 143,612 & 143,667 & $0.35 \%$ & $0.39 \%$ & $0.42 \%$ \\
\hline 15 & 6 & 1 & 5 & $\infty$ & 0.7 & 100,754 & 1.4 & 101,046 & 101,105 & 101,350 & $0.29 \%$ & $0.35 \%$ & $0.59 \%$ \\
\hline 15 & 6 & 1 & 5 & $\infty$ & 0.95 & 31,885 & $<1$ & 31,901 & 31,909 & 31,918 & $0.05 \%$ & $0.08 \%$ & $0.11 \%$ \\
\hline 20 & 1 & 1 & 5 & 2 & 0.7 & 355,451 & 6.5 & 355,842 & 355,920 & 356,061 & $0.11 \%$ & $0.13 \%$ & $0.17 \%$ \\
\hline 20 & 1 & 1 & 5 & 2 & 0.95 & 355,451 & 6.4 & 356,304 & 356,731 & 358,010 & $0.24 \%$ & $0.36 \%$ & $0.72 \%$ \\
\hline 20 & 1 & 1 & 5 & 5 & 0.7 & 113,597 & 2.5 & 113,801 & 113,924 & 114,251 & $0.18 \%$ & $0.29 \%$ & $0.58 \%$ \\
\hline 20 & 1 & 1 & 5 & 5 & 0.95 & 113597 & 2.4 & 113,733 & 113,815 & 114,033 & $0.12 \%$ & $0.19 \%$ & $0.38 \%$ \\
\hline 20 & 1 & 1 & 5 & $\infty$ & 0.7 & 13661 & $<1$ & 13,672 & 13,673 & 13,680 & $0.08 \%$ & $0.09 \%$ & $0.14 \%$ \\
\hline 20 & 1 & 1 & 5 & $\infty$ & 0.95 & 13661 & $<1$ & 13,661 & 13,683 & 13,703 & $0.00 \%$ & $0.16 \%$ & $0.30 \%$ \\
\hline 20 & 3 & 1 & 5 & 2 & 0.7 & 510207 & 238.8 & 514,085 & 514,472 & 514,899 & $0.76 \%$ & $0.84 \%$ & $0.92 \%$ \\
\hline 20 & 3 & 1 & 5 & 2 & 0.95 & 510,207 & 214.3 & 515,207 & 515,707 & 518,457 & $0.98 \%$ & $1.08 \%$ & $1.62 \%$ \\
\hline 20 & 3 & 1 & 5 & 5 & 0.7 & 155,869 & 29.4 & 157,163 & 157,551 & 158,728 & $0.83 \%$ & $1.08 \%$ & $1.83 \%$ \\
\hline 20 & 3 & 1 & 5 & 5 & 0.95 & 155,869 & 31.3 & 157,225 & 157,361 & 158,554 & $0.87 \%$ & $0.96 \%$ & $1.72 \%$ \\
\hline 20 & 3 & 1 & 5 & $\infty$ & 0.7 & 37,090 & $<1$ & 37,327 & 37,375 & 37,460 & $0.64 \%$ & $0.77 \%$ & $1.00 \%$ \\
\hline 20 & 3 & 1 & 5 & $\infty$ & 0.95 & 22,250 & $<1$ & 22,370 & 22,418 & 22,452 & $0.54 \%$ & $0.76 \%$ & $0.91 \%$ \\
\hline 20 & 6 & 1 & 5 & 2 & 0.7 & 602,010 & $1,968.5$ & 607,247 & 610,390 & 615,418 & $0.87 \%$ & $1.39 \%$ & $2.23 \%$ \\
\hline 20 & 6 & 1 & 5 & 2 & 0.95 & 602,010 & $1,299.4$ & 606,826 & 609,234 & 612,124 & $0.80 \%$ & $1.20 \%$ & $1.68 \%$ \\
\hline 20 & 6 & 1 & 5 & 5 & 0.7 & 200,509 & 214.1 & 201,772 & 202,404 & 204,299 & $0.63 \%$ & $0.95 \%$ & $1.89 \%$ \\
\hline 20 & 6 & 1 & 5 & 5 & 0.95 & 200,509 & 238.7 & 202,334 & 202,881 & 205,253 & $0.91 \%$ & $1.18 \%$ & $2.37 \%$ \\
\hline 20 & 6 & 1 & 5 & $\infty$ & 0.7 & 95,198 & 3.1 & 95,712 & 96,021 & 96,350 & $0.54 \%$ & $0.86 \%$ & $1.21 \%$ \\
\hline 20 & 6 & 1 & 5 & $\infty$ & 0.95 & 31,885 & $<1$ & 31,965 & 32,013 & 32,038 & $0.25 \%$ & $0.40 \%$ & $0.48 \%$ \\
\hline 25 & 1 & 1 & 5 & 2 & 0.7 & 471,281 & 35.4 & 472,930 & 473,260 & 474,052 & $0.35 \%$ & $0.42 \%$ & $0.59 \%$ \\
\hline 25 & 1 & 1 & 5 & 2 & 0.95 & 471,281 & 33.5 & 473,213 & 473,406 & 473,619 & $0.41 \%$ & $0.45 \%$ & $0.50 \%$ \\
\hline 25 & 1 & 1 & 5 & 5 & 0.7 & 127,433 & 21.1 & 127,803 & 127,987 & 128,542 & $0.29 \%$ & $0.44 \%$ & $0.87 \%$ \\
\hline 25 & 1 & 1 & 5 & 5 & 0.95 & 127,433 & 18.7 & 127,930 & 128,079 & 128,531 & $0.39 \%$ & $0.51 \%$ & $0.86 \%$ \\
\hline 25 & 1 & 1 & 5 & $\infty$ & 0.7 & 13661 & $<1$ & 13,717 & 13,745 & 13,795 & $0.41 \%$ & $0.62 \%$ & $0.98 \%$ \\
\hline 25 & 1 & 1 & 5 & $\infty$ & 0.95 & 13,661 & $<1$ & 13,709 & 13,728 & 13,741 & $0.35 \%$ & $0.49 \%$ & $0.59 \%$ \\
\hline 25 & 3 & 1 & 5 & 2 & 0.7 & 585,441 & 771.1 & 589,305 & 589,691 & 592,667 & $0.66 \%$ & $0.73 \%$ & $1.23 \%$ \\
\hline 25 & 3 & 1 & 5 & 2 & 0.95 & 585,441 & 601.9 & 590,183 & 590,657 & 595,352 & $0.81 \%$ & $0.89 \%$ & $1.69 \%$ \\
\hline 25 & 3 & 1 & 5 & 5 & 0.7 & 172,858 & 101.1 & 174,189 & 174,721 & 176,585 & $0.77 \%$ & $1.08 \%$ & $2.16 \%$ \\
\hline 25 & 3 & 1 & 5 & 5 & 0.95 & 172,858 & 88.0 & 173,791 & 174,071 & 174,557 & $0.54 \%$ & $0.70 \%$ & $0.98 \%$ \\
\hline 25 & 3 & 1 & 5 & $\infty$ & 0.7 & 37,090 & $<1$ & 37,220 & 37,285 & 37,460 & $0.35 \%$ & $0.53 \%$ & $1.00 \%$ \\
\hline 25 & 3 & 1 & 5 & $\infty$ & 0.95 & 21,720 & $<1$ & 21,783 & 21,802 & 21,859 & $0.29 \%$ & $0.38 \%$ & $0.64 \%$ \\
\hline 25 & 6 & 1 & 5 & 2 & 0.7 & 748,191 & $15,628.0$ & 757,020 & 760,551 & 767,967 & $1.18 \%$ & $1.65 \%$ & $2.64 \%$ \\
\hline 25 & 6 & 1 & 5 & 2 & 0.95 & 74,8191 & $6,351.3$ & 757,693 & 763,394 & 772,516 & $1.27 \%$ & $2.03 \%$ & $3.25 \%$ \\
\hline 25 & 6 & 1 & 5 & 5 & 0.7 & 246,657 & $1,579.4$ & 249,395 & 249,669 & 250,271 & $1.11 \%$ & $1.22 \%$ & $1.47 \%$ \\
\hline 25 & 6 & 1 & 5 & 5 & 0.95 & 246,657 & 802.0 & 248,877 & 249,765 & 251,319 & $0.90 \%$ & $1.26 \%$ & $1.89 \%$ \\
\hline 25 & 6 & 1 & 5 & $\infty$ & 0.7 & 95,964 & 3.9 & 96,713 & 96,862 & 97,491 & $0.78 \%$ & $0.94 \%$ & $1.59 \%$ \\
\hline 25 & 6 & 1 & 5 & $\infty$ & 0.95 & 31,885 & $<1$ & 32,064 & 32,153 & 32,314 & $0.56 \%$ & $0.84 \%$ & $1.34 \%$ \\
\hline
\end{tabular}


Table 6: Results for the instance with $(\alpha, \beta)=(2,5)$

\begin{tabular}{|c|c|c|c|c|c|c|c|c|c|c|c|c|c|}
\hline \multirow{2}{*}{$|N|$} & \multirow{2}{*}{$|T|$} & \multirow{2}{*}{$\alpha$} & \multirow{2}{*}{$\beta$} & \multirow{2}{*}{$\bar{\lambda}$} & \multirow{2}{*}{$\pi$} & \multicolumn{2}{|l|}{ Gurobi } & \multicolumn{3}{|l|}{ Heuristic } & \multicolumn{3}{|l|}{ Gap } \\
\hline & & & & & & Objective & Time (s) & Best & Average & Worst & Best & Average & Worst \\
\hline 15 & 1 & 2 & 5 & 2 & 0.7 & 334,751 & 3.2 & 334,751 & 335,287 & 335,769 & $0.00 \%$ & $0.16 \%$ & $0.30 \%$ \\
\hline 15 & 1 & 2 & 5 & 2 & 0.95 & 334751 & 3.2 & 334,952 & 335,119 & 335,340 & $0.06 \%$ & $0.11 \%$ & $0.18 \%$ \\
\hline 15 & 1 & 2 & 5 & 5 & 0.7 & 97552 & $<1$ & 97,552 & 97,718 & 97,884 & $0.00 \%$ & $0.17 \%$ & $0.34 \%$ \\
\hline 15 & 1 & 2 & 5 & 5 & 0.95 & 97,552 & $<1$ & 97,581 & 97,757 & 97,798 & $0.03 \%$ & $0.21 \%$ & $0.25 \%$ \\
\hline 15 & 1 & 2 & 5 & $\infty$ & 0.7 & 13,661 & $<1$ & 13,661 & 13,705 & 13,718 & $0.00 \%$ & $0.32 \%$ & $0.42 \%$ \\
\hline 15 & 1 & 2 & 5 & $\infty$ & 0.95 & 13,661 & $<1$ & 13,661 & 13,683 & 13,692 & $0.00 \%$ & $0.16 \%$ & $0.22 \%$ \\
\hline 15 & 3 & 2 & 5 & 2 & 0.7 & 418,084 & 77.0 & 419,756 & 420,258 & 422,215 & $0.40 \%$ & $0.52 \%$ & $0.99 \%$ \\
\hline 15 & 3 & 2 & 5 & 2 & 0.95 & 418084 & 74.6 & 419,046 & 419,526 & 420,680 & $0.23 \%$ & $0.35 \%$ & $0.62 \%$ \\
\hline 15 & 3 & 2 & 5 & 5 & 0.7 & 129,164 & 6.7 & 129,913 & 129,988 & 130,730 & $0.58 \%$ & $0.64 \%$ & $1.21 \%$ \\
\hline 15 & 3 & 2 & 5 & 5 & 0.95 & 129,164 & 8.8 & 129,513 & 129,548 & 129,855 & $0.27 \%$ & $0.30 \%$ & $0.53 \%$ \\
\hline 15 & 3 & 2 & 5 & $\infty$ & 0.7 & 52,040 & $<1$ & 52,212 & 52,280 & 52,521 & $0.33 \%$ & $0.46 \%$ & $0.92 \%$ \\
\hline 15 & 3 & 2 & 5 & $\infty$ & 0.95 & 22559 & $<1$ & 22,654 & 22,682 & 22,719 & $0.42 \%$ & $0.55 \%$ & $0.71 \%$ \\
\hline 15 & 6 & 2 & 5 & 2 & 0.7 & 504,970 & 652.1 & 509,717 & 510,666 & 512,944 & $0.94 \%$ & $1.13 \%$ & $1.58 \%$ \\
\hline 15 & 6 & 2 & 5 & 2 & 0.95 & 504,970 & 518.6 & 509,363 & 509,803 & 512,702 & $0.87 \%$ & $0.96 \%$ & $1.53 \%$ \\
\hline 15 & 6 & 2 & 5 & 5 & 0.7 & 206,586 & 39.0 & 207,949 & 208,359 & 208,890 & $0.66 \%$ & $0.86 \%$ & $1.12 \%$ \\
\hline 15 & 6 & 2 & 5 & 5 & 0.95 & 175,112 & 62.8 & 176,355 & 177,101 & 178,295 & $0.71 \%$ & $1.14 \%$ & $1.82 \%$ \\
\hline 15 & 6 & 2 & 5 & $\infty$ & 0.7 & 155,336 & 4.2 & 155,724 & 155,841 & 156,144 & $0.25 \%$ & $0.33 \%$ & $0.52 \%$ \\
\hline 15 & 6 & 2 & 5 & $\infty$ & 0.95 & 47,475 & $<1$ & 47,689 & 47,774 & 47,924 & $0.45 \%$ & $0.63 \%$ & $0.95 \%$ \\
\hline 20 & 1 & 2 & 5 & 2 & 0.7 & 457,064 & 20.3 & 457,795 & 458,234 & 458,936 & $0.16 \%$ & $0.26 \%$ & $0.41 \%$ \\
\hline 20 & 1 & 2 & 5 & 2 & 0.95 & 457,064 & 19.0 & 457,064 & 458,070 & 458,572 & $0.00 \%$ & $0.22 \%$ & $0.33 \%$ \\
\hline 20 & 1 & 2 & 5 & 5 & 0.7 & 133,322 & 4.0 & 133,735 & 133,777 & 134,095 & $0.31 \%$ & $0.34 \%$ & $0.58 \%$ \\
\hline 20 & 1 & 2 & 5 & 5 & 0.95 & 133,322 & 3.1 & 133,469 & 133,483 & 133,516 & $0.11 \%$ & $0.12 \%$ & $0.15 \%$ \\
\hline 20 & 1 & 2 & 5 & $\infty$ & 0.7 & 13,661 & $<1$ & 13,661 & 13,686 & 13,710 & $0.00 \%$ & $0.18 \%$ & $0.36 \%$ \\
\hline 20 & 1 & 2 & 5 & $\infty$ & 0.95 & 13,661 & $<1$ & 13,661 & 13,673 & 13,676 & $0.00 \%$ & $0.09 \%$ & $0.11 \%$ \\
\hline 20 & 3 & 2 & 5 & 2 & 0.7 & 562,675 & $1,128.3$ & 568,921 & 570,170 & 576,915 & $1.11 \%$ & $1.33 \%$ & $2.53 \%$ \\
\hline 20 & 3 & 2 & 5 & 2 & 0.95 & 562,675 & $1,042.1$ & 566,783 & 567,604 & 569,576 & $0.73 \%$ & $0.88 \%$ & $1.23 \%$ \\
\hline 20 & 3 & 2 & 5 & 5 & 0.7 & 163,021 & 48.5 & 164,456 & 164,599 & 164,757 & $0.88 \%$ & $0.97 \%$ & $1.06 \%$ \\
\hline 20 & 3 & 2 & 5 & 5 & 0.95 & 163,021 & 39.2 & 164,081 & 164,187 & 164,536 & $0.65 \%$ & $0.72 \%$ & $0.93 \%$ \\
\hline 20 & 3 & 2 & 5 & $\infty$ & 0.7 & 63,447 & 1.6 & 63,821 & 63,934 & 64,420 & $0.59 \%$ & $0.77 \%$ & $1.53 \%$ \\
\hline 20 & 3 & 2 & 5 & $\infty$ & 0.95 & 22,559 & $<1$ & 22,609 & 22,624 & 22,662 & $0.22 \%$ & $0.29 \%$ & $0.46 \%$ \\
\hline 20 & 6 & 2 & 5 & 2 & 0.7 & 563,738 & $12,028.7$ & 569,432 & 572,848 & 574,670 & $1.01 \%$ & $1.62 \%$ & $1.94 \%$ \\
\hline 20 & 6 & 2 & 5 & 2 & 0.95 & 579,115 & $10,571.0$ & 584,559 & 586 , & 592,071 & $0.94 \%$ & $1.32 \%$ & $2.24 \%$ \\
\hline 20 & 6 & 2 & 5 & 5 & 0.7 & 248,071 & 498.2 & 250,328 & 251,006 & 251,593 & $0.91 \%$ & $1.18 \%$ & $1.42 \%$ \\
\hline 20 & 6 & 2 & 5 & 5 & 0.95 & 225,091 & 933.5 & 227,049 & 227,637 & 228,146 & $0.87 \%$ & $1.13 \%$ & $1.36 \%$ \\
\hline 20 & 6 & 2 & 5 & $\infty$ & 0.7 & 169325 & 4.5 & 170,561 & 171,056 & 171,402 & $0.73 \%$ & $1.02 \%$ & $1.23 \%$ \\
\hline 20 & 6 & 2 & 5 & $\infty$ & 0.95 & 53805 & 1.4 & 54,106 & 54,287 & 54,335 & $0.56 \%$ & $0.90 \%$ & $0.99 \%$ \\
\hline 25 & 1 & 2 & 5 & 2 & 0.7 & 544,986 & 101.8 & 549,237 & 550,512 & 554933 & $0.78 \%$ & $1.01 \%$ & $1.83 \%$ \\
\hline 25 & 1 & 2 & 5 & 2 & 0.95 & 544,986 & 96.9 & 548,310 & 549,640 & 550,571 & $0.61 \%$ & $0.85 \%$ & $1.02 \%$ \\
\hline 25 & 1 & 2 & 5 & 5 & 0.7 & 161,749 & 32.7 & 162,218 & 162,453 & 162,945 & $0.29 \%$ & $0.44 \%$ & $0.74 \%$ \\
\hline 25 & 1 & 2 & 5 & 5 & 0.95 & 161,749 & 21.1 & 162,445 & 162,862 & 163,196 & $0.43 \%$ & $0.69 \%$ & $0.89 \%$ \\
\hline 25 & 1 & 2 & 5 & $\infty$ & 0.7 & 13,661 & $<1$ & 13,731 & 13,772 & 13,806 & $0.51 \%$ & $0.82 \%$ & $1.06 \%$ \\
\hline 25 & 1 & 2 & 5 & $\infty$ & 0.95 & 13,661 & $<1$ & 13,697 & 13,718 & 13,746 & $0.26 \%$ & $0.42 \%$ & $0.62 \%$ \\
\hline 25 & 3 & 2 & 5 & 2 & 0.7 & 652,828 & 973.9 & 658,638 & 659,800 & 665,378 & $0.89 \%$ & $1.07 \%$ & $1.92 \%$ \\
\hline 25 & 3 & 2 & 5 & 2 & 0.95 & 652,828 & 2358.6 & 659,813 & 660,512 & 661,280 & $1.07 \%$ & $1.18 \%$ & $1.29 \%$ \\
\hline 25 & 3 & 2 & 5 & 5 & 0.7 & 197,837 & 230.2 & 199,420 & 199,895 & 200,718 & $0.80 \%$ & $1.04 \%$ & $1.46 \%$ \\
\hline 25 & 3 & 2 & 5 & 5 & 0.95 & 197,837 & 212.0 & 199,776 & 200,745 & 202,490 & $0.98 \%$ & $1.47 \%$ & $2.35 \%$ \\
\hline 25 & 3 & 2 & 5 & $\infty$ & 0.7 & 56,428 & 1.1 & 56,857 & 56,943 & 57,046 & $0.76 \%$ & $0.91 \%$ & $1.09 \%$ \\
\hline 25 & 3 & 2 & 5 & $\infty$ & 0.95 & 21720 & $<1$ & 21,824 & 21,887 & 21,987 & $0.48 \%$ & $0.77 \%$ & $1.23 \%$ \\
\hline 25 & 6 & 2 & 5 & 2 & 0.7 & 761,326 & $18,000.0$ & 770,462 & 773,203 & 783,892 & $1.20 \%$ & $1.56 \%$ & $2.96 \%$ \\
\hline 25 & 6 & 2 & 5 & 2 & 0.95 & 799570 & $18,000.0$ & 807,406 & 808,189 & 815,947 & $0.98 \%$ & $1.08 \%$ & $2.05 \%$ \\
\hline 25 & 6 & 2 & 5 & 5 & 0.7 & 278,346 & $2,220.0$ & 282,521 & 285,026 & 287,030 & $1.50 \%$ & $2.40 \%$ & $3.12 \%$ \\
\hline 25 & 6 & 2 & 5 & 5 & 0.95 & 272,862 & $3,222.0$ & 275,209 & 276,382 & 279,198 & $0.86 \%$ & $1.29 \%$ & $2.32 \%$ \\
\hline 25 & 6 & 2 & 5 & $\infty$ & 0.7 & 165,051 & 13.5 & 166,355 & 166,876 & 167,059 & $0.79 \%$ & $1.11 \%$ & $1.22 \%$ \\
\hline 25 & 6 & 2 & 5 & $\infty$ & 0.95 & 48,569 & 1.7 & 48,865 & 48,984 & 49,274 & $0.61 \%$ & $0.85 \%$ & $1.45 \%$ \\
\hline
\end{tabular}


Table 7: Results for the instance with $(\alpha, \beta)=(5,5)$

\begin{tabular}{|c|c|c|c|c|c|c|c|c|c|c|c|c|c|}
\hline \multirow{2}{*}{$|N|$} & \multirow{2}{*}{$|T|$} & \multirow{2}{*}{$\alpha$} & \multirow{2}{*}{$\beta$} & \multirow{2}{*}{$\bar{\lambda}$} & \multirow{2}{*}{$\pi$} & \multicolumn{2}{|l|}{ Gurobi } & \multicolumn{3}{|l|}{ Heuristic } & \multicolumn{3}{|l|}{ Gap } \\
\hline & & & & & & Objective & Time (s) & Best & Average & Worst & Best & Average & Worst \\
\hline 15 & 1 & 5 & 5 & 2 & 0.7 & 285,263 & 3.6 & 285,263 & 285,691 & 285,905 & $0.00 \%$ & $0.15 \%$ & $0.23 \%$ \\
\hline 15 & 1 & 5 & 5 & 2 & 0.95 & 285,263 & 3.5 & 285,263 & 285,862 & 286,102 & $0.00 \%$ & $0.21 \%$ & $0.29 \%$ \\
\hline 15 & 1 & 5 & 5 & 5 & 0.7 & 87,198 & $<1$ & 87,198 & 87,338 & 87,463 & $0.00 \%$ & $0.16 \%$ & $0.30 \%$ \\
\hline 15 & 1 & 5 & 5 & 5 & 0.95 & 87,198 & $<1$ & 87,198 & 87,451 & 87,577 & $0.00 \%$ & $0.29 \%$ & $0.44 \%$ \\
\hline 15 & 1 & 5 & 5 & $\infty$ & 0.7 & 13,661 & $<1$ & 13,661 & 13,686 & 13,700 & $0.00 \%$ & $0.18 \%$ & $0.29 \%$ \\
\hline 15 & 1 & 5 & 5 & $\infty$ & 0.95 & 13,661 & $<1$ & 13,661 & 13,681 & 13,684 & $0.00 \%$ & $0.15 \%$ & $0.17 \%$ \\
\hline 15 & 3 & 5 & 5 & 2 & 0.7 & 364,987 & 50.1 & 365,790 & 365,870 & 366,224 & $0.22 \%$ & $0.24 \%$ & $0.34 \%$ \\
\hline 15 & 3 & 5 & 5 & 2 & 0.95 & 364,987 & 47.2 & 366,848 & 367,965 & 368,859 & $0.51 \%$ & $0.82 \%$ & $1.06 \%$ \\
\hline 15 & 3 & 5 & 5 & 5 & 0.7 & 113,337 & 3.7 & 113,700 & 113,736 & 113,856 & $0.32 \%$ & $0.35 \%$ & $0.46 \%$ \\
\hline 15 & 3 & 5 & 5 & 5 & 0.95 & 113,337 & 4.5 & 114,006 & 114,407 & 115,263 & $0.59 \%$ & $0.94 \%$ & $1.70 \%$ \\
\hline 15 & 3 & 5 & 5 & $\infty$ & 0.7 & 51,731 & $<1$ & 51,731 & 51,819 & 51,898 & $0.00 \%$ & $0.17 \%$ & $0.32 \%$ \\
\hline 15 & 3 & 5 & 5 & $\infty$ & 0.95 & 22,250 & $<1$ & 22,299 & 22,304 & 22,352 & $0.22 \%$ & $0.24 \%$ & $0.46 \%$ \\
\hline 15 & 6 & 5 & 5 & 2 & 0.7 & 455,523 & 275.6 & 458,621 & 459,860 & 463,329 & $0.68 \%$ & $0.95 \%$ & $1.71 \%$ \\
\hline 15 & 6 & 5 & 5 & 2 & 0.95 & 455,523 & 244.7 & 460,169 & 461,563 & 465,791 & $1.02 \%$ & $1.33 \%$ & $2.25 \%$ \\
\hline 15 & 6 & 5 & 5 & 5 & 0.7 & 179,837 & 19.9 & 181,204 & 182,024 & 183,336 & $0.76 \%$ & $1.22 \%$ & $1.95 \%$ \\
\hline 15 & 6 & 5 & 5 & 5 & 0.95 & 157,942 & 40.5 & 158,511 & 158,567 & 158,630 & $0.36 \%$ & $0.40 \%$ & $0.44 \%$ \\
\hline 15 & 6 & 5 & 5 & $\infty$ & 0.7 & 138,651 & 3.3 & 139,053 & 139,174 & 139,383 & $0.29 \%$ & $0.38 \%$ & $0.53 \%$ \\
\hline 15 & 6 & 5 & 5 & $\infty$ & 0.95 & 47,475 & 1.3 & 47,632 & 47,663 & 47,851 & $0.33 \%$ & $0.40 \%$ & $0.79 \%$ \\
\hline 20 & 1 & 5 & 5 & 2 & 0.7 & 359,325 & 23.6 & 359,936 & 360,302 & 361,084 & $0.17 \%$ & $0.27 \%$ & $0.49 \%$ \\
\hline 20 & 1 & 5 & 5 & 2 & 0.95 & 359,325 & 21.9 & 360,439 & 360,996 & 361,330 & $0.31 \%$ & $0.47 \%$ & $0.56 \%$ \\
\hline 20 & 1 & 5 & 5 & 5 & 0.7 & 106,636 & 2.3 & 106,636 & 107,180 & 107,343 & $0.00 \%$ & $0.51 \%$ & $0.66 \%$ \\
\hline 20 & 1 & 5 & 5 & 5 & 0.95 & 106,636 & 2.2 & 106,945 & 107,069 & 107,156 & $0.29 \%$ & $0.41 \%$ & $0.49 \%$ \\
\hline 20 & 1 & 5 & 5 & $\infty$ & 0.7 & 13,661 & $<1$ & 13,661 & 13,691 & 13,697 & $0.00 \%$ & $0.22 \%$ & $0.26 \%$ \\
\hline 20 & 1 & 5 & 5 & $\infty$ & 0.95 & 13,661 & $<1$ & 13,676 & 13,681 & 13,700 & $0.11 \%$ & $0.14 \%$ & $0.29 \%$ \\
\hline 20 & 3 & 5 & 5 & 2 & 0.7 & 469,628 & 415.5 & 473,996 & 474,869 & 479,586 & $0.93 \%$ & $1.12 \%$ & $2.12 \%$ \\
\hline 20 & 3 & 5 & 5 & 2 & 0.95 & 469,628 & 454.4 & 474,935 & 475,996 & 479,817 & $1.13 \%$ & $1.36 \%$ & $2.17 \%$ \\
\hline 20 & 3 & 5 & 5 & 5 & 0.7 & 156,222 & 43.0 & 157,769 & 158,697 & 160,429 & $0.99 \%$ & $1.58 \%$ & $2.69 \%$ \\
\hline 20 & 3 & 5 & 5 & 5 & 0.95 & 156,222 & 44.0 & 157,831 & 158,475 & 160,277 & $1.03 \%$ & $1.44 \%$ & $2.60 \%$ \\
\hline 20 & 3 & 5 & 5 & $\infty$ & 0.7 & 56,571 & $<1$ & 57,148 & 57,379 & 57,460 & $1.02 \%$ & $1.43 \%$ & $1.57 \%$ \\
\hline 20 & 3 & 5 & 5 & $\infty$ & 0.95 & 22,250 & $<1$ & 22,399 & 22,459 & 22,584 & $0.67 \%$ & $0.94 \%$ & $1.50 \%$ \\
\hline 20 & 6 & 5 & 5 & 2 & 0.7 & 576,945 & $10,812.0$ & 583,118 & 584,970 & 589,785 & $1.07 \%$ & $1.39 \%$ & $2.23 \%$ \\
\hline 20 & 6 & 5 & 5 & 2 & 0.95 & 619,430 & $9,358.8$ & 625,129 & 627 & 630,600 & $0.92 \%$ & $1.29 \%$ & $1.80 \%$ \\
\hline 20 & 6 & 5 & 5 & 5 & 0.7 & 205,960 & 136.0 & 207,855 & 208,992 & 210,508 & $0.92 \%$ & $1.47 \%$ & $2.21 \%$ \\
\hline 20 & 6 & 5 & 5 & 5 & 0.95 & 196,848 & 268.4 & 199,033 & 199,470 & 201,568 & $1.11 \%$ & $1.33 \%$ & $2.40 \%$ \\
\hline 20 & 6 & 5 & 5 & $\infty$ & 0.7 & 145,360 & 4.9 & 146,755 & 147,314 & 148,290 & $0.96 \%$ & $1.34 \%$ & $2.02 \%$ \\
\hline 20 & 6 & 5 & 5 & $\infty$ & 0.95 & 37,114 & $<1$ & 37,337 & 37,470 & 37,684 & $0.60 \%$ & $0.96 \%$ & $1.54 \%$ \\
\hline 25 & 1 & 5 & 5 & 2 & 0.7 & 451,850 & 28.2 & 454,245 & 455,203 & 455,538 & $0.53 \%$ & $0.74 \%$ & $0.82 \%$ \\
\hline 25 & 1 & 5 & 5 & 2 & 0.95 & 451,850 & 27.8 & 453,567 & 453,910 & 455,971 & $0.38 \%$ & $0.46 \%$ & $0.91 \%$ \\
\hline 25 & 1 & 5 & 5 & 5 & 0.7 & 122,717 & 6.7 & 123,318 & 123,499 & 123,655 & $0.49 \%$ & $0.64 \%$ & $0.76 \%$ \\
\hline 25 & 1 & 5 & 5 & 5 & 0.95 & 122,717 & 6.9 & 123,257 & 123,527 & 123,851 & $0.44 \%$ & $0.66 \%$ & $0.92 \%$ \\
\hline 25 & 1 & 5 & 5 & $\infty$ & 0.7 & 13,661 & $<1$ & 13,692 & 13,708 & 13,727 & $0.23 \%$ & $0.35 \%$ & $0.48 \%$ \\
\hline 25 & 1 & 5 & 5 & $\infty$ & 0.95 & 13661 & $<1$ & 13,661 & 13,707 & 13,740 & $0.00 \%$ & $0.34 \%$ & $0.58 \%$ \\
\hline 25 & 3 & 5 & 5 & 2 & 0.7 & 573124 & 759.6 & 578970 & 582477 & 583,413 & $1.02 \%$ & $1.63 \%$ & $1.80 \%$ \\
\hline 25 & 3 & 5 & 5 & 2 & 0.95 & 573,124 & 706.8 & 580,976 & 581,761 & 585,216 & $1.37 \%$ & $1.51 \%$ & $2.11 \%$ \\
\hline 25 & 3 & 5 & 5 & 5 & 0.7 & 164,402 & 87.0 & 166,128 & 167,164 & 169,374 & $1.05 \%$ & $1.68 \%$ & $3.02 \%$ \\
\hline 25 & 3 & 5 & 5 & 5 & 0.95 & 164,402 & 108.7 & 166,720 & 168,111 & 170,173 & $1.41 \%$ & $2.26 \%$ & $3.51 \%$ \\
\hline 25 & 3 & 5 & 5 & $\infty$ & 0.7 & 51,201 & $<1$ & 51,892 & 52,100 & 52,639 & $1.35 \%$ & $1.76 \%$ & $2.81 \%$ \\
\hline 25 & 3 & 5 & 5 & $\infty$ & 0.95 & 21,720 & $<1$ & 21,957 & 22,075 & 22,288 & $1.09 \%$ & $1.64 \%$ & $2.62 \%$ \\
\hline 25 & 6 & 5 & 5 & 2 & 0.7 & 698,295 & $18,000.0$ & 707,233 & 710,809 & 723,322 & $1.28 \%$ & $1.79 \%$ & $3.58 \%$ \\
\hline 25 & 6 & 5 & 5 & 2 & 0.95 & 695,576 & $18,000.0$ & 702,045 & 703,985 & 707,349 & $0.93 \%$ & $1.21 \%$ & $1.69 \%$ \\
\hline 25 & 6 & 5 & 5 & 5 & 0.7 & 245,205 & $1,905.9$ & 247,583 & 248,297 & 250,771 & $0.97 \%$ & $1.26 \%$ & $2.27 \%$ \\
\hline 25 & 6 & 5 & 5 & 5 & 0.95 & 225,201 & 629.8 & 227,791 & 228,568 & 231,598 & $1.15 \%$ & $1.50 \%$ & $2.84 \%$ \\
\hline 25 & 6 & 5 & 5 & $\infty$ & 0.7 & 150,292 & 9.6 & 151,705 & 152,411 & 154,106 & $0.94 \%$ & $1.41 \%$ & $2.54 \%$ \\
\hline 25 & 6 & 5 & 5 & $\infty$ & 0.95 & 37,114 & 1.2 & 37,426 & 37,457 & 37,697 & $0.84 \%$ & $0.92 \%$ & $1.57 \%$ \\
\hline
\end{tabular}


Table 8: Results of $|T|=1$

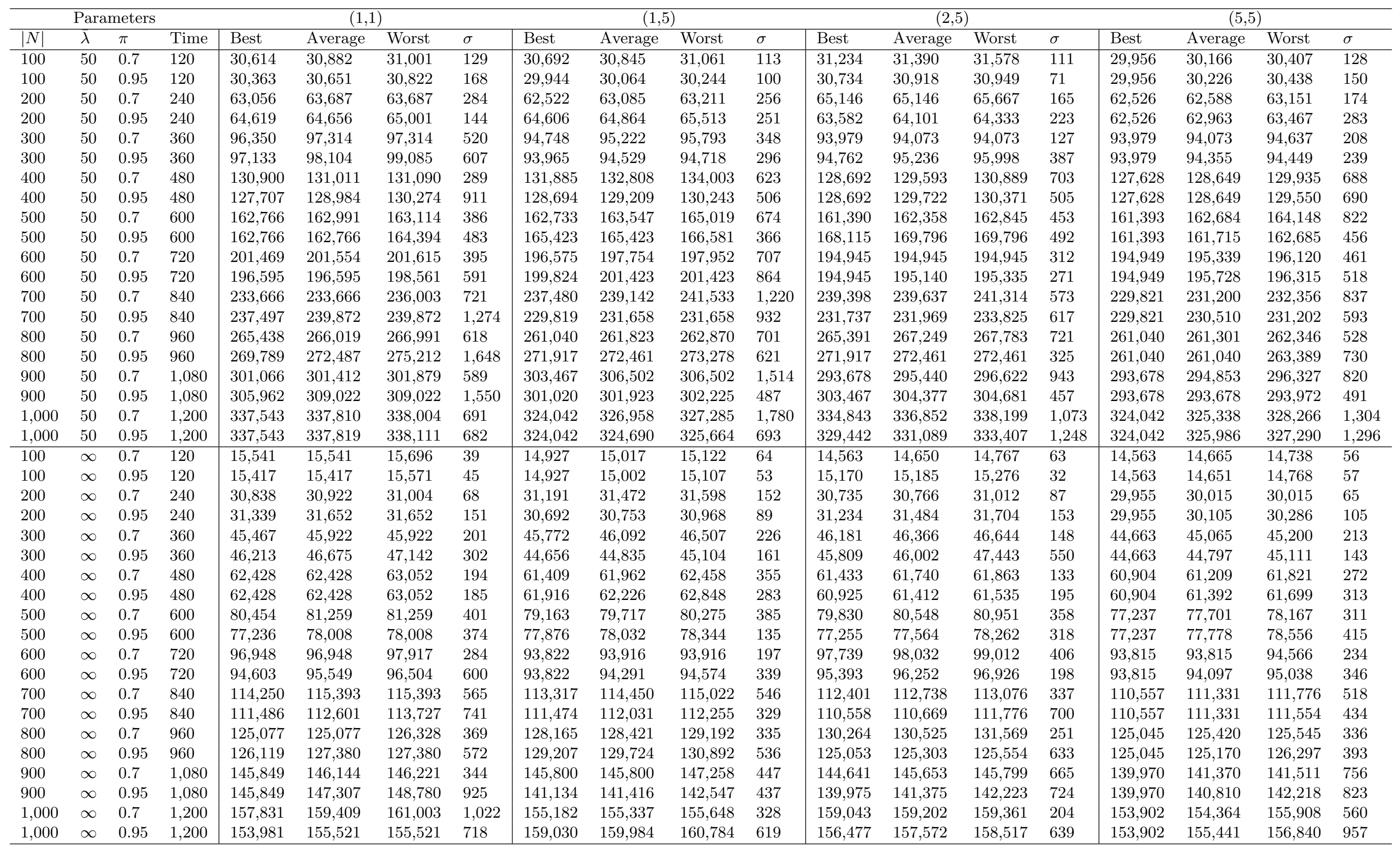


Table 9: Results of $|T|=3$

\begin{tabular}{|c|c|c|c|c|c|c|c|c|c|c|c|c|c|c|c|c|c|c|c|}
\hline \multicolumn{4}{|c|}{ Parameters } & \multicolumn{4}{|c|}{$(1,1)$} & \multicolumn{4}{|c|}{$(1,5)$} & \multicolumn{4}{|c|}{$(2,5)$} & \multicolumn{4}{|c|}{$(5,5)$} \\
\hline$\overline{|N|}$ & $\bar{\lambda}$ & $\pi$ & Time & Best & Average & Worst & $\sigma$ & Best & Average & Worst & $\sigma$ & Best & Average & Worst & $\sigma$ & Best & Average & Worst & $\sigma$ \\
\hline 100 & 50 & 0.7 & 360 & 177,495 & 177,691 & 177,995 & 378 & 42,511 & 42,639 & 42,852 & 111 & 59,880 & 60,359 & 60,419 & 166 & 60,212 & 60,573 & 60,815 & 211 \\
\hline 100 & 50 & 0.95 & 360 & 44,017 & 44,718 & 45,002 & 348 & 43,208 & 43,251 & 43,554 & 110 & 42,870 & 43,170 & 43,386 & 159 & 41,814 & 42,148 & 42,232 & 140 \\
\hline 200 & 50 & 0.7 & 720 & 195,994 & 196,006 & 196,054 & 258 & 89,951 & 90,041 & 90,941 & 285 & 93,747 & 94,403 & 94,875 & 344 & 89,953 & 90,492 & 91,306 & 420 \\
\hline 200 & 50 & 0.95 & 720 & 89,968 & 90,868 & 90,868 & 392 & 93,699 & 94,542 & 95,109 & 474 & 90,747 & 91,654 & 92,021 & 400 & 89,953 & 90,043 & 90,673 & 229 \\
\hline 300 & 50 & 0.7 & 1,080 & 201,084 & 203,095 & 205,126 & 1,240 & 132,860 & 133,657 & 134,994 & 742 & 135,083 & 135,488 & 136,843 & 539 & 131,767 & 132,952 & 133,484 & 620 \\
\hline 300 & 50 & 0.95 & 1,080 & 132,904 & 134,233 & 134,233 & 735 & 131,762 & 132,948 & 134,012 & 727 & 136,181 & 136,181 & 136,317 & 179 & 131,767 & 132,821 & 132,954 & 614 \\
\hline 400 & 50 & 0.7 & 1,440 & 207,841 & 209,919 & 212,018 & 1,357 & 179,017 & 179,554 & 180,811 & 610 & 181,958 & 183,232 & 183,415 & 457 & 176,073 & 177,481 & 178,546 & 1,017 \\
\hline 400 & 50 & 0.95 & 1,440 & 179,097 & 180,888 & 182,697 & 1,107 & 176,082 & 176,258 & 176,963 & 308 & 181,958 & 183,596 & 184,698 & 861 & 176,073 & 177,481 & 179,078 & 973 \\
\hline 500 & 50 & 0.95 & 1,800 & 228,068 & 228,068 & 230,349 & 669 & 229,880 & 230,340 & 232,643 & 787 & 233,653 & 234,120 & 234,822 & 363 & 226,093 & 226,997 & 227,678 & 574 \\
\hline 600 & 50 & 0.7 & 2,160 & 277,407 & 280,181 & 282,983 & 1,475 & 288,885 & 290,329 & 290,329 & 810 & 286,572 & 286,859 & 288,293 & 540 & 277,307 & 279,526 & 281,762 & 1,522 \\
\hline 600 & 50 & 0.95 & 2,160 & 279,719 & 280,009 & 281,445 & 560 & 281,951 & 283,361 & 284,778 & 900 & 277,328 & 278,160 & 279,273 & 534 & 277,307 & 278,971 & 281,203 & 1,272 \\
\hline 700 & 50 & 0.7 & 2,520 & 333,356 & 336,690 & 340,057 & 2,047 & 330,569 & 332,883 & 334,547 & 1,45 & 327,828 & 327,828 & 328,811 & 461 & 327,811 & 328,467 & 330,438 & 970 \\
\hline 700 & 50 & 0.95 & 2,520 & 327,891 & 331,170 & 334,482 & 1,889 & 327,837 & 328,493 & 330,792 & 871 & 338,756 & 341,466 & 342,149 & 992 & 327,811 & 328,139 & 330,108 & 733 \\
\hline 800 & 50 & 0.7 & 2,880 & 385,240 & 389,092 & 392,983 & 2,685 & 372,706 & 376,060 & 377,940 & 1,705 & 382,024 & 382,944 & 383,003 & 490 & 372,706 & 374,570 & 378,316 & 1,627 \\
\hline 800 & 50 & 0.95 & 2,880 & 385,240 & 386,104 & 386,267 & 974 & 375,812 & 376,564 & 379,200 & 1,189 & 372,706 & 372,706 & 374,942 & 744 & 372,706 & 375,315 & 377,942 & 1,871 \\
\hline 900 & 50 & 0.7 & 3,240 & 428,295 & 432,578 & 432,578 & 2,287 & 421,262 & 423,368 & 426,755 & 1,698 & 421,262 & 422,105 & 423,793 & 795 & 417,780 & 418,198 & 420,289 & 892 \\
\hline 900 & 50 & 0.95 & 3,240 & 424,813 & 429,061 & 433,352 & 2,720 & 428,225 & 432,079 & 435,104 & 2,623 & 417,780 & 419,869 & 421,129 & 1,071 & 417,780 & 420,705 & 421,126 & 1,810 \\
\hline 1,000 & 50 & 0.95 & 3,600 & 462,451 & 462,881 & 463,014 & 1,124 & 462,451 & 462,913 & 466,153 & 1,159 & 470,095 & 473,386 & 477,173 & 2,228 & 458,629 & 460,005 & 462,765 & 1,438 \\
\hline 100 & $\infty$ & 0.7 & 360 & $\begin{array}{l}178,962 \\
\end{array}$ & 180,752 & 182,560 & 1,141 & 36,982 & 37,315 & 37,688 & 206 & 55,441 & 55,829 & 56,052 & 199 & 54,428 & 54,972 & 55,137 & 224 \\
\hline 100 & $\infty$ & 0.95 & 360 & 32,981 & 33,311 & 33,644 & 202 & 21,217 & 21,387 & 21,473 & 85 & 21,371 & 21,521 & 21,672 & 98 & 20,533 & 20,697 & 20,863 & 109 \\
\hline 200 & $\infty$ & 0.7 & 720 & 183,653 & 183,910 & 184,005 & 257 & 44,434 & 44,656 & 44,969 & 180 & 57,748 & 58,037 & 58,559 & 246 & 57,205 & 57,720 & 57,893 & 230 \\
\hline 200 & $\infty$ & 0.95 & 720 & 43,536 & 43,781 & 44,001 & 181 & 42,732 & 42,817 & 42,903 & 79 & 42,798 & 42,884 & 43,013 & 72 & 41,365 & 41,614 & 41,739 & 147 \\
\hline 300 & $\infty$ & 0.7 & 1,080 & 183,093 & 184,924 & 186,773 & 1,106 & 63,593 & 64,165 & 64,486 & 299 & 67,244 & 67,782 & 67,850 & 190 & 63,749 & 64,195 & 64,644 & 251 \\
\hline 300 & $\infty$ & 0.95 & 1,080 & 65,171 & 65,823 & 66,481 & 382 & 65,170 & 65,561 & 65,561 & 191 & 63,105 & 63,484 & 63,611 & 146 & 63,077 & 63,266 & 63,899 & 240 \\
\hline 400 & $\infty$ & 0.7 & 1,440 & 181,499 & 183,314 & 185,147 & 1,076 & 87,312 & 87,574 & 88,362 & 324 & 88,141 & 88,758 & 89,024 & 281 & 84,517 & 85,109 & 85,109 & 283 \\
\hline 400 & $\infty$ & 0.95 & 1,440 & 85,917 & 86,776 & 87,644 & 511 & 87,312 & 87,399 & 87,923 & 201 & 86,631 & 87,044 & 80,101 & 2,403 & 84,502 & 84,840 & 84,925 & 226 \\
\hline 500 & $\infty$ & 0.7 & 1,800 & 178,678 & 179,004 & 179,412 & 327 & 110,178 & 111,059 & 111,836 & 627 & 110,196 & 111,188 & 111,744 & 483 & 107,495 & 107,925 & 108,788 & 443 \\
\hline 500 & $\infty$ & 0.95 & 1,800 & 111,085 & 112,196 & 112,196 & 467 & 110,178 & 110,729 & 110,950 & 413 & 111,988 & 112,996 & 113,561 & 504 & 107,495 & 108,140 & 108,248 & 329 \\
\hline 600 & $\infty$ & 0.7 & 2,160 & 194,695 & 194,695 & 196,642 & 556 & 135,264 & 136,211 & 137,028 & 569 & 134,171 & 134,708 & 135,920 & 553 & 130,876 & 131,007 & 131,400 & 269 \\
\hline 600 & $\infty$ & 0.95 & 2,160 & 130,951 & 132,261 & 133,584 & 738 & 131,992 & 132,388 & 133,712 & 513 & 133,080 & 134,145 & 134,145 & 347 & 130,876 & 131,662 & 132,847 & 623 \\
\hline 700 & $\infty$ & 0.7 & 2,520 & 199,823 & 200,005 & 200,112 & 448 & 155,346 & 155,346 & 156,744 & 419 & 157,918 & 158,708 & 159,978 & 645 & 154,049 & 155,589 & 156,678 & 859 \\
\hline 700 & $\infty$ & 0.95 & 2,520 & 160,545 & 162,150 & 163,772 & 1,067 & 156,630 & 156,630 & 156,943 & 247 & 154,066 & 154,066 & 155,607 & 443 & 154,049 & 155,435 & 156,368 & 790 \\
\hline 800 & $\infty$ & 0.7 & 2,880 & 214,751 & 216,899 & 216,899 & 1,139 & 184,061 & 184,981 & 185,906 & 686 & 182,595 & 183,508 & 184,426 & 552 & 176,690 & 177,221 & 178,816 & 562 \\
\hline 800 & $\infty$ & 0.95 & 2,880 & 182,668 & 182,921 & 183,056 & 364 & 181,116 & 181,297 & 182,747 & 504 & 182,595 & 182,960 & 184,424 & 590 & 176,690 & 176,867 & 177,044 & 324 \\
\hline 900 & $\infty$ & 0.7 & 3,240 & 223,364 & 225,598 & 227,854 & 1,443 & 198,950 & 199,149 & 199,348 & 338 & 202,230 & 203,848 & 204,256 & 640 & 197,292 & 199,265 & 199,464 & 889 \\
\hline
\end{tabular}


Table 10: Results of $|T|=6$

\begin{tabular}{|c|c|c|c|c|c|c|c|c|c|c|c|c|c|c|c|c|c|c|c|}
\hline \multicolumn{4}{|c|}{ Parameters } & \multicolumn{4}{|c|}{$(1,1)$} & \multicolumn{4}{|c|}{$(1,5)$} & \multicolumn{4}{|c|}{$(2,5)$} & \multicolumn{4}{|c|}{$(5,5)$} \\
\hline$|N|$ & $\bar{\lambda}$ & $\pi$ & Time & Best & Average & Worst & $\sigma$ & Best & Average & Worst & $\sigma$ & Best & Average & Worst & $\sigma$ & Best & Average & Worst & $\sigma$ \\
\hline 100 & 50 & 0.7 & 720 & 463,597 & 468,233 & 468,233 & 1,907 & 122,803 & 123,908 & 123,908 & 505 & 167,433 & 167,768 & 168,104 & 275 & 156,238 & 157,332 & 157,961 & 651 \\
\hline 100 & 50 & 0.95 & 720 & 102,487 & 102,487 & 103,512 & 324 &, 582 & 64,969 & 65,229 & 222 & 4,056 & 64,056 & 64,633 & 171 & 63,528 & 63,972 & 64,612 & 321 \\
\hline 200 & 50 & 0.7 & 1,440 & 461,913 & 466,532 & 471,197 & 2,871 & 160,358 & 161,641 & 163,096 & 786 & 184,818 & 185,557 & 185,928 & 324 & 177,408 & 179,005 & 180,795 & 971 \\
\hline 200 & 50 & 0.95 & 1,440 & 131,786 & 131,919 & 132,102 & 220 & 137,235 & 37,235 & 137,784 & 213 & 134,015 & 135,087 & 136,168 & 693 & 131,763 & 132,949 & 134,146 & 773 \\
\hline 300 & 50 & 0.7 & 2,160 & 431,989 & 431,989 & 436,309 & 1,345 & 202,787 & 203,801 & 205,024 & 727 & 213,900 & 215,397 & 217,120 & 1,038 & 197,314 & 199,090 & 200,285 & 1,087 \\
\hline 300 & 50 & 0.95 & 2,160 & 191,956 & 193,876 & 193,876 & 924 & 195,072 & 195,072 & 195,852 & 297 & 188,906 & 190,417 & 190,607 & 519 & 187,285 & 188,221 & 189,915 & 726 \\
\hline 400 & 50 & 0.7 & 2,880 & 460,056 & 464,657 & 469,304 & 543 & 262,665 & 264,241 & 264,241 & 825 & 262,480 & 263,005 & 264,846 & 717 & 253,873 & 255,650 & 257,951 & 1,177 \\
\hline 400 & 50 & 0.95 & 2,880 & 256,059 & 258,620 & 258,620 & 517 & 258,115 & 258,373 & 258,631 & 418 & 264,498 & 266,349 & 268,213 & 1,169 & 253,873 & 253,873 & 255,142 & 514 \\
\hline 500 & 50 & 0.7 & 3,600 & 483,055 & 487,886 & 487,886 & 2,219 & 323,750 & 324,398 & 326,669 & 941 & 331 , & 335,038 & 337 & & 318 & 734 & & 849 \\
\hline 500 & 50 & 0.95 & 3,600 & 329, & 329, & 332 & 944 & 318, & 319 & 321 & 897 & 2 & 31 & 32 & 962 & 31 & 90 & 32 & 1,342 \\
\hline 600 & 50 & 0.7 & 4,320 & 510 & 510,566 & 515,672 & 480 & 397,019 & 399,004 & 401,797 & 1,769 & 406,765 & 410,019 & 410,839 & 1,265 & 390,462 & 391,243 & 391,634 & 902 \\
\hline 600 & 50 & 0.95 & 4,320 & 390,548 & 390,548 & 394,453 & 154 & 393,764 & 394,552 & 397,708 & 1,192 & 397,003 & 400,973 & 404,582 & 2,439 & 390,462 & 393,195 & 61 & .526 \\
\hline 700 & 50 & 0.7 & 040 & 543 & 545,171 & & & 1 & 28 & 48 & 1,071 & 46 & 18 & & 19 & 26 & 118 & & 1,216 \\
\hline 700 & 50 & 0.9 & 040 & & 36 & & & 9 & 33 & & 2 & & & & & & 35 & & \\
\hline 800 & 50 & 0.7 &, 760 & & 55 & & & 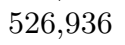 & & & 2 & & & & & & 400 & & \\
\hline 800 & 50 & 0.9 & 5,760 & 74 & 43 & & & & & & & & & & & & 271 & & 2,695 \\
\hline 900 & 50 & 0.7 & 6,480 & 02 & 632 & & & & 560 & & 2, & & & & 727 & & 716 & & 2,519 \\
\hline 900 & 50 & 0.95 & 480 & 37 & 612 & & & 607 & 29 & 609 & 907 & & 26 & & 2,505 & & 763 & & 2,733 \\
\hline 1,000 & 50 & 0.7 & 7,200 & 8 & 10 & & & & & & 935 & & & & & & & & 2,520 \\
\hline 1,000 & 50 & 0.95 & 7,200 & 645,270 & 645,661 & 645 & 1,258 & 650 , & 657,065 & 657 & 2,680 & 639 & 901 & 647,036 & 2,180 & 634,692 & 231 & & 2,192 \\
\hline 100 & $\infty$ & 0.7 & 720 & 459,797 & 464,395 & 464,395 & 1,711 & 117,446 & 117,798 & 118,976 & 442 & 161,263 & 161,263 & 161,586 & 182 & 154,391 & 154,855 & 155,165 & 389 \\
\hline 100 & $\infty$ & 0.95 & 720 & 8,910 & 99,002 & 99,441 & & 30,735 & 30,981 & 31,074 & 153 & & 32,195 & & 44 & & & & 57 \\
\hline 200 & $\infty$ & 0.7 & 1,440 & 457,000 & 457,000 & 461,570 & 1,264 & 123,393 & 124,504 & 125,500 & 681 & & & & 587 & & 152,452 & & 348 \\
\hline 200 & $\infty$ & 0.95 & 1,440 & 98,278 & 98,278 & 99,261 & & 64,507 & 65,088 & & 307 & 63,479 & 63,923 & & & & & & 212 \\
\hline 300 & $\infty$ & 0.7 & & & & & & & & & 2 & & & & & & 152,221 & & 7 \\
\hline 300 & $\infty$ & 0. & & & & & & & & & & & & & & & & & \\
\hline 400 & $\infty$ & 0. & & & & & & & & & & & & & & & & & \\
\hline 400 & $\infty$ & 0. & & & & & & 2 & & & & & & & & & & & \\
\hline 500 & $\infty$ & 0 & 2000 & & & & & 6 & & 17 & 2 & & & & 27 & & 495 & & 720 \\
\hline 500 & $\infty$ & 0. & . & & & & 44 & & & & $x$ & & & & 48 & & 823 & 23 & 722 \\
\hline 600 & $\infty$ & 0.7 & 4,320 & 79 & 88 & 43 & & 20 & 86 & & 32 & 60 & & & 73 & & 37 & 20 & 458 \\
\hline 600 & $\infty$ & 0.95 & 4,320 & 81 & 192,891 & 1 & 74 & 189,426 & 26 & 190 & 436 & 86 & 89 & & & 184 & & & 416 \\
\hline 700 & $\infty$ & 0.7 & 5,040 & & 450,701 & & & & & & & & & & 45 & & & & 742 \\
\hline 700 & $\infty$ & 0.95 & 5,040 & 8,945 & 221,134 & 221,134 & & 200,001 & 101 & 203 & 1,09 & & 630 & & 90 & & 998 & 164 & 927 \\
\hline 800 & $\infty$ & 0.7 & 5,760 & 445,925 & 446,111 & 446,925 & 788 & 212,389 & 212,601 & 214,514 & 659 & 214,102 & 215,815 & 217,757 & 1,143 & 205,538 & 205,538 & 206,360 & 346 \\
\hline 800 & $\infty$ & 0.95 & 5,760 & 236,510 & 238,875 & 241,264 & 1,329 & 206,102 & 206,102 & 207,751 & 464 & 199,453 & 200,450 & 200,450 & 354 & 199,453 & 201,049 & 201,853 & 783 \\
\hline 900 & $\infty$ & 0.7 & 6,480 & 436,947 & 441,316 & 441,316 & 2,195 & 208,593 & 209,427 & 210,893 & 800 & 93 & 209,219 & 209,428 & 330 & 206,869 & 208,731 & 209,148 & 1,029 \\
\hline 900 & $\infty$ & 0.95 & 6,480 & 246,977 & 246,977 & 249,447 & 722 & 206,401 & 207,020 & 207,434 & 534 & 93 & 209,342 & 209,761 & 472 & 203,017 & 203,423 & 204,847 & 530 \\
\hline 1,000 & $\infty$ & 0 . & 7,200 & 442,0 & 446 & & & 210 , & 212, & 214 & 1,08 & & & 21 & 47 & 208 & 209,771 & 209 & 482 \\
\hline 1,000 & $\infty$ & 0.95 & 7,200 & 265,695 & 265,891 & 266,012 & 536 & 214,036 & 215,962 & 216,178 & 1,013 & 208,900 & 209,109 & 210,782 & 589 & 205,475 & 207,324 & 208,153 & 885 \\
\hline
\end{tabular}


Table 11: Results of the Beasley [70] instances

\begin{tabular}{|c|c|c|c|c|c|c|c|c|c|c|c|c|c|c|c|c|}
\hline \multirow{2}{*}{ Instance } & \multirow[b]{2}{*}{$|N|$} & \multicolumn{5}{|c|}{$|T|=1$} & \multicolumn{5}{|c|}{$|T|=3$} & \multicolumn{5}{|c|}{$|T|=6$} \\
\hline & & Time & Best & Average & Worst & $\sigma$ & Time & Best & Average & Worst & $\sigma$ & Time & Best & Average & Worst & $\sigma$ \\
\hline pmed1 & 100 & 120 & 113,777 & 114,573 & 115,490 & 547 & 360 & $1,113,922$ & $1,115,036$ & $1,120,611$ & 1,829 & 720 & $2,621,262$ & $2,626,505$ & $2,629,132$ & 2,608 \\
\hline pmed2 & 100 & 120 & 107,862 & 108,941 & 109,921 & 878 & 360 & $1,055,433$ & $1,056,488$ & $1,061,770$ & 1,866 & 720 & $2,433,800$ & $2,436,234$ & $2,441,106$ & 2,515 \\
\hline pmed3 & 100 & 120 & 112,493 & 113,280 & 114,413 & 714 & 360 & $1,132,450$ & $1,134,715$ & $1,139,254$ & 2,029 & 720 & $2,679,723$ & $2,682,403$ & $2,687,768$ & 2,950 \\
\hline pmed4 & 100 & 120 & 110,465 & 110,686 & 111,682 & 400 & 360 & $1,126,159$ & $1,129,537$ & $1,134,055$ & 2,485 & 720 & $2,714,670$ & $2,720,099$ & $2,725,539$ & 3,633 \\
\hline pmed5 & 100 & 120 & 98,460 & 98,755 & 99,644 & 378 & 360 & 852,209 & 853,061 & 856,473 & 1,244 & 720 & $2,278,011$ & $2,282,567$ & $2,284,850$ & 2,162 \\
\hline pmed6 & 200 & 240 & 165,888 & 167,547 & 168,050 & 755 & 720 & $1,630,259$ & $1,635,150$ & $1,640,055$ & 2,912 & 1,440 & $3,737,267$ & $3,741,004$ & $3,748,486$ & 3,718 \\
\hline pmed7 & 200 & 240 & 141,719 & 143,136 & 143,852 & 876 & 720 & $1,512,401$ & $1,515,426$ & $1,519,972$ & 2,459 & 1,440 & $3,761,620$ & $3,769,143$ & $3,776,681$ & 4,628 \\
\hline pmed8 & 200 & 240 & 137,589 & 138,965 & 139,660 & 736 & 720 & $1,588,046$ & $1,591,222$ & $1,594,404$ & 2,227 & 1,440 & $3,964,318$ & $3,968,282$ & $3,976,219$ & 4,096 \\
\hline pmed9 & 200 & 240 & 162,155 & 162,804 & 163,130 & 390 & 720 & $1,480,788$ & $1,483,750$ & $1,488,201$ & 2,356 & 1,440 & $3,762,916$ & $3,766,679$ & $3,770,446$ & 3,073 \\
\hline pmed10 & 200 & 240 & 127,704 & 128,215 & 129,369 & 612 & 720 & $1,140,789$ & $1,141,930$ & $1,145,356$ & 1,518 & 1,440 & $3,194,135$ & $3,200,523$ & $3,206,924$ & 3,930 \\
\hline pmed11 & 300 & 360 & 141,719 & 143,136 & 143,422 & 526 & 1,080 & $1,455,797$ & $1,457,253$ & $1,460,168$ & 1,421 & 2,160 & $3,932,233$ & $3,940,097$ & $3,947,977$ & 4,977 \\
\hline pmed12 & 300 & 360 & 146,026 & 146,902 & 148,224 & 774 & 1,080 & $1,386,920$ & $1,391,081$ & $1,395,254$ & 2,560 & 2,160 & $3,823,420$ & $3,827,243$ & $3,834,897$ & 3,515 \\
\hline pmed13 & 300 & 360 & 148,690 & 149,285 & 149,584 & 266 & 1,080 & $1,363,244$ & $1,367,334$ & $1,370,069$ & 1,892 & 2,160 & $4,040,619$ & $4,044,660$ & $4,052,749$ & 4,020 \\
\hline pmed14 & 300 & 360 & 163,332 & 164,475 & 165,133 & 626 & 1,080 & $1,375,588$ & $1,378,339$ & $1,381,096$ & 1,839 & 2,160 & $3,906,544$ & $3,910,451$ & $3,914,361$ & 4,118 \\
\hline pmed15 & 300 & 360 & 151,639 & 152,397 & 153,159 & 504 & 1,080 & $1,337,956$ & $1,339,294$ & $1,343,312$ & 1,730 & 2,160 & $3,849,205$ & $3,853,054$ & $3,860,760$ & 3,539 \\
\hline pmed16 & 400 & 480 & 156,984 & 158,397 & 159,031 & 668 & 1,440 & 925,736 & 926,662 & 928,515 & 863 & 2,880 & $2,967,213$ & $2,973,147$ & $2,979,093$ & 3,909 \\
\hline pmed17 & 400 & 480 & 145,961 & 147,275 & 148,306 & 715 & 1,440 & 859,030 & 859,889 & 864,188 & 1,546 & 2,880 & $2,810,868$ & $2,813,679$ & $2,819,306$ & 3,095 \\
\hline pmed18 & 400 & 480 & 155,194 & 155,660 & 157,217 & 636 & 1,440 & $1,247,759$ & $1,250,255$ & $1,255,256$ & 2,287 & 2,880 & $3,499,936$ & $3,503,436$ & $3,506,939$ & 2,882 \\
\hline pmed19 & 400 & 480 & 151,259 & 151,562 & 152,623 & 449 & 1,440 & 940,258 & 943,079 & 945,908 & 1,542 & 2,880 & $3,075,437$ & $3,078,512$ & $3,084,669$ & 3,177 \\
\hline pmed20 & 400 & 480 & 159,126 & 160,399 & 161,843 & 895 & 1,440 & $1,016,720$ & $1,017,737$ & $1,020,790$ & 1,475 & 2,880 & $3,054,630$ & $3,057,685$ & $3,060,743$ & 2,495 \\
\hline pmed21 & 500 & 600 & 178,223 & 179,114 & 180,547 & 405 & 1,800 & 631,814 & 632,446 & 634,976 & 918 & 3,600 & $2,137,946$ & $2,140,084$ & $2,144,364$ & 2,254 \\
\hline pmed22 & 500 & 600 & 182,250 & 183,708 & 185,361 & 889 & 1,800 & 809,235 & 811,663 & 815,721 & 1,893 & 3,600 & $2,629,539$ & $2,634,798$ & $2,640,068$ & 3,329 \\
\hline pmed23 & 500 & 600 & 176,327 & 177,914 & 179,337 & 918 & 1,800 & 772,260 & 773,032 & 774,578 & 823 & 3,600 & $2,572,788$ & $2,575,361$ & $2,580,512$ & 2,560 \\
\hline pmed24 & 500 & 600 & 177,973 & 179,575 & 181,191 & 961 & 1,800 & 724,235 & 725,683 & 727,860 & 1,051 & 3,600 & $2,296,600$ & $2,298,897$ & $2,301,196$ & 1,937 \\
\hline pmed25 & 500 & 600 & 184,306 & 184,675 & 186,522 & 818 & 1,800 & 765,699 & 767,996 & 769,532 & 1,125 & 3,600 & $2,552,448$ & $2,557,553$ & $2,560,111$ & 2,467 \\
\hline
\end{tabular}




\section{References}

[1] Office of National Statistics. Healthcare expenditure in the UK, 2015.

[2] Preventive health care, what's the problem. http://www.cdc.gov/healthcommunication/ toolstemplates/entertainmented/tips/preventivehealth.html, 2013.

[3] Gianfranco Damiani, Bruno Federico, Danila Basso, Alessandra Ronconi, Caterina Bianca Neve Aurora Bianchi, Gian Marco Anzellotti, Gabriella Nasi, Franco Sassi, and Walter Ricciardi. Socioeconomic disparities in the uptake of breast and cervical cancer screening in Italy: a cross sectional study. BMC Public Health, 12(1):1, 2012.

[4] Jared B Fox and Frederic E Shaw. Clinical preventive services coverage and the affordable care act. American Journal of Public Health, 105(1):e7-e10, 2015.

[5] Helen I Meissner, Nancy Breen, Carrie N Klabunde, and Sally W Vernon. Patterns of colorectal cancer screening uptake among men and women in the United States. Cancer Epidemiology Biomarkers 86 Prevention, 15(2):389-394, 2006.

[6] Rita Santos, Hugh Gravelle, and Carol Propper. Does quality affect patients choice of doctor? evidence from England. The Economic Journal, 127(600):445-494, 2016.

[7] Ivo Muler, Tom Smith, Steve Mellor, Lawrence Rare, and Blaise Genton. The effect of distance from home on attendance at a small rural health centre in Papua New Guinea. International Journal of Epidemiology, 27(5):878-884, 1998.

[8] Marco Varkevisser, Stéphanie A van der Geest, and Frederik T Schut. Do patients choose hospitals with high quality ratings? empirical evidence from the market for angioplasty in the Netherlands. Journal of Health Economics, 31(2):371-378, 2012.

[9] Robin Haynes, Andrew Lovett, and Gisela Sunnenberg. Potential accessibility, travel time, and consumer choice: geographical variations in general medical practice registrations in eastern England. Environment and Planning A, 35(10):1733-1750, 2003.

[10] Harold Hotelling. Stability in competition. In The Collected Economics Articles of Harold Hotelling, pages 50-63. Springer, 1990.

[11] Masao Nakanishi and Lee G Cooper. Parameter estimation for a multiplicative competitive interaction model: least squares approach. Journal of Marketing Research, 11(3):303-311, 1974.

[12] David R Bell, Teck-Hua Ho, and Christopher S Tang. Determining where to shop: Fixed and variable costs of shopping. Journal of Marketing Research, 35(3):352-369, 1998.

[13] Oded Berman, Zvi Drezner, and Dmitry Krass. Cooperative cover location problems: the planar case. IIE Transactions, 42(3):232-246, 2009.

[14] S Louis Hakimi. Optimum locations of switching centers and the absolute centers and medians of a graph. Operations Research, 12(3):450-459, 1964.

[15] Dong-Guen Kim and Yeong-Dae Kim. A lagrangian heuristic algorithm for a public healthcare facility location problem. Annals of Operations Research, 206(1):221-240, 2013.

[16] Siddhartha S Syam and Murray J Côté. A location-allocation model for service providers with application to not-for-profit health care organizations. Omega, 38(3):157-166, 2010.

[17] Marjolein Veenstra, Kees Jan Roodbergen, Leandro C Coelho, and Stuart X Zhu. A simultaneous facility location and vehicle routing problem arising in health care logistics in the Netherlands. August 2016(CIRRELT-2016-44).

[18] Amir Ahmadi-Javid, Pardis Seyedi, and Siddhartha S Syam. A survey of healthcare facility location. Computers \& Operations Research, 79:223-263, 2017.

[19] Vedat Verter and Sophie D Lapierre. Location of preventive health care facilities. Annals of Operations Research, 110(1-4):123-132, 2002. 
[20] Yue Zhang, Oded Berman, and Vedat Verter. Incorporating congestion in preventive healthcare facility network design. European Journal of Operational Research, 198(3):922-935, 2009.

[21] Yue Zhang, Oded Berman, Patrice Marcotte, and Vedat Verter. A bilevel model for preventive healthcare facility network design with congestion. IIE Transactions, 42(12):865-880, 2010.

[22] Wei Gu, Xin Wang, and Elizabeth S. McGregor . Optimization of preventive health care facility locations. International Journal of Health Geographics, 9(1):17, 2010.

[23] Yue Zhang, Oded Berman, and Vedat Verter. The impact of client choice on preventive healthcare facility network design. OR Spectrum, 34(2):349-370, 2012.

[24] Soheil Davari, Kemal Kilic, and Gurdal Ertek. Fuzzy bi-objective preventive health care network design. Health Care Management Science, 18(3):303-317, 2015.

[25] Knut Haase and Sven Müller. Insights into clients choice in preventive health care facility location planning. OR Spectrum, 37(1):273-291, 2015.

[26] Soheil Davari, Kemal Kilic, and Siamak Naderi. A heuristic approach to solve the preventive health care problem with budget and congestion constraints. Applied Mathematics and Computation, 276:442-453, 2016.

[27] Mitsuo Gen and Admi Syarif. Hybrid genetic algorithm for multi-time period production/distribution planning. Computers \& Industrial Engineering, 48(4):799-809, 2005.

[28] Alan R McKendall Jr and Jin Shang. Hybrid ant systems for the dynamic facility layout problem. Computers 63 Operations Research, 33(3):790-803, 2006.

[29] Hyun Jeung Ko and Gerald W Evans. A genetic algorithm-based heuristic for the dynamic integrated forward/reverse logistics network for 3PLs. Computers $\&$ Operations Research, 34(2):346-366, 2007.

[30] Wei Yi and Linet Özdamar. A dynamic logistics coordination model for evacuation and support in disaster response activities. European Journal of Operational Research, 179(3):1177-1193, 2007.

[31] Malick Ndiaye and Hesham Alfares. Modeling health care facility location for moving population groups. Computers \& Operations Research, 35(7):2154-2161, 2008.

[32] Xue-Feng Wang, Xiao-Ming Sun, and Yang Fang. Genetic algorithm solution for multi-period two-echelon integrated competitive/uncompetitive facility location problem. Asia-Pacific Journal of Operational Research, 25(01):33-56, 2008.

[33] Hari K Rajagopalan, Cem Saydam, and Jing Xiao. A multiperiod set covering location model for dynamic redeployment of ambulances. Computers \& Operations Research, 35(3):814-826, 2008.

[34] Riccardo Manzini and Elisa Gebennini. Optimization models for the dynamic facility location and allocation problem. International Journal of Production Research, 46(8):2061-2086, 2008.

[35] Éric Gourdin and Olivier Klopfenstein. Multi-period capacitated location with modular equipments. Computers $\&$ Operations Research, 35(3):661-682, 2008.

[36] Yolanda Hinojosa, Jörg Kalcsics, Stefan Nickel, Justo Puerto, and Sebastian Velten. Dynamic supply chain design with inventory. Computers $\&$ Operations Research, 35(2):373-391, 2008.

[37] Maria Albareda-Sambola, Elena Fernandez, Yolanda Hinojosa, and Justo Puerto. The multi-period incremental service facility location problem. Computers $\&$ Operations Research, 36(5):1356-1375, 2009.

[38] Der-Horng Lee and Meng Dong. Dynamic network design for reverse logistics operations under uncertainty. Transportation Research Part E: Logistics and Transportation Review, 45(1):61-71, 2009.

[39] Stephen Mahar, Kurt M Bretthauer, and MA Venkataramanan. An algorithm for solving the multi-period online fulfillment assignment problem. Mathematical and Computer Modelling, 50(9):1294-1304, 2009.

[40] Verena Schmid and Karl F Doerner. Ambulance location and relocation problems with time-dependent travel times. European Journal of Operational Research, 207(3):1293-1303, 2010. 
[41] Ayfer Basar, Bulent Catay, and Tonguc Unluyurt. A multi-period double coverage approach for locating the emergency medical service stations in Istanbul. Journal of the Operational Research Society, 62(4):627-637, 2011.

[42] Mohammad Hossein Fazel Zarandi, Soheil Davari, and Ali Haddad Sisakht. The large scale maximal covering location problem. Scientia Iranica, 18(6):1564-1570, 2011.

[43] Joaquin E Torres-Soto and Halit Üster. Dynamic-demand capacitated facility location problems with and without relocation. International Journal of Production Research, 49(13):3979-4005, 2011.

[44] James C Benneyan, Hande Musdal, Mehmet Erkan Ceyhan, Brian Shiner, and Bradley V Watts. Specialty care single and multi-period location-allocation models within the veterans health administration. Socioeconomic Planning Sciences, 46(2):136-148, 2012.

[45] Yue Sha and Jun Huang. The multi-period location-allocation problem of engineering emergency blood supply systems. Systems Engineering Procedia, 5:21-28, 2012.

[46] Beate Rottkemper, Kathrin Fischer, and Alexander Blecken. A transshipment model for distribution and inventory relocation under uncertainty in humanitarian operations. Socio-Economic Planning Sciences, 46(1):98 - 109, 2012.

[47] Verena Schmid. Solving the dynamic ambulance relocation and dispatching problem using approximate dynamic programming. European Journal of Operational Research, 219(3):611-621, 2012.

[48] Maria Albareda-Sambola, Elena Fernández, and Stefan Nickel. Multiperiod location-routing with decoupled time scales. European Journal of Operational Research, 217(2):248-258, 2012.

[49] Maria Albareda-Sambola, Antonio Alonso-Ayuso, Laureano F Escudero, Elena Fernández, and Celeste Pizarro. Fix-and-relax-coordination for a multi-period location-allocation problem under uncertainty. Computers $\&$ Operations Research, 40(12):2878-2892, 2013.

[50] Abdolsalam Ghaderi and Mohammad Saeed Jabalameli. Modeling the budget-constrained dynamic uncapacitated facility location-network design problem and solving it via two efficient heuristics: a case study of health care. Mathematical and Computer Modelling, 57(3):382-400, 2013.

[51] Francisco Saldanha-da-Gama Isabel Correia, Teresa Melo. Comparing classical performance measures for a multi-period, two-echelon supply chain network design problem with sizing decisions. Computers \& Industrial Engineering, 64:366-380, 2012.

[52] Lu Zhen, Kai Wang, Hongtao Hu, and Daofang Chang. A simulation optimization framework for ambulance deployment and relocation problems. Computers $\&$ Industrial Engineering, 72(0):12 - 23, 2014.

[53] Shahin Gelareh, Rahimeh Neamatian Monemi, and Stefan Nickel. Multi-period hub location problems in transportation. Transportation Research Part E: Logistics and Transportation Review, 75:67 - 94, 2015.

[54] Sung Hoon Chung and Changhyun Kwon. Multi-period planning for electric car charging station locations: A case of Korean expressways. European Journal of Operational Research, 242(2):677-687, 2015.

[55] Maria Elbek and Sanne Wøhlk. A variable neighborhood search for the multi-period collection of recyclable materials. European Journal of Operational Research, 249(2):540-550, 2016.

[56] Christophe Duhamel, Andréa Cynthia Santos, Daniel Brasil, Eric Châtelet, and Babiga Birregah. Connecting a population dynamic model with a multi-period location-allocation problem for post-disaster relief operations. Annals of Operations Research, 247(2):1-21, 2016.

[57] Isabel Correia and Teresa Melo. Multi-period capacitated facility location under delayed demand satisfaction. European Journal of Operational Research, 255(3):729 - 746, 2016.

[58] Nikola Markovic, Ilya O. Ryzhov, and Paul Schonfeld. Evasive flow capture: A multi-period stochastic facility location problem with independent demand. European Journal of Operational Research, 257(2):687 $-703,2017$.

[59] Amit Kumar Vatsa and Sachin Jayaswal. A new formulation and benders decomposition for the multiperiod maximal covering facility location problem with server uncertainty. European Journal of Operational Research, 251(2):404 - 418, 2016. 
[60] Jesus T Pastor. Bicriterion programs and managerial location decisions: application to the banking sector. Journal of the Operational Research Society, 45(12):1351-1362, 1994.

[61] Tammy Drezner. Derived attractiveness of shopping malls. IMA Journal of Management Mathematics, 17(4):349-358, 2006.

[62] Tammy Drezner. A review of competitive facility location in the plane. Logistics Research, 7(1):1-12, 2014.

[63] William John Reilly. The law of retail gravitation. New York: Knickerbocker Press, 1931.

[64] Nenad Mladenović and Pierre Hansen. Variable neighborhood search. Computers 83 Operations Research, 24(11):1097-1100, 1997.

[65] Hamid Karimi, Seyed Habib A Rahmati, and M Zandieh. An efficient knowledge-based algorithm for the flexible job shop scheduling problem. Knowledge-Based Systems, 36:236-244, 2012.

[66] Slim Belhaiza, Pierre Hansen, and Gilbert Laporte. A hybrid variable neighborhood tabu search heuristic for the vehicle routing problem with multiple time windows. Computers $\& 3$ Operations Research, 52:269-281, 2014 .

[67] Soheil Davari, Mohammad Hossein Fazel Zarandi, and I Burhan Turksen. A greedy variable neighborhood search heuristic for the maximal covering location problem with fuzzy coverage radii. Knowledge-Based Systems, 41:68-76, 2013.

[68] Pierre Hansen, Nenad Mladenović, and José A Moreno Pérez. Variable neighbourhood search: methods and applications. 4OR, 6(4):319-360, 2008.

[69] Mika Sato and Lakhmi C Jain. Innovations in Fuzzy Clustering: Theory and Applications (Studies in Fuzziness and Soft Computing). Springer-Verlag, 2006.

[70] John E Beasley. A note on solving large p-median problems. European Journal of Operational Research, 21(2):270-273, 1985. 